M. Fukushima

Nagoya Math. J.

Vol. 74 (1979), 137-168

\title{
A DECOMPOSITION OF ADDITIVE FUNCTIONALS OF FINITE ENERGY
}

\author{
MASATOSHI FUKUSHIMA
}

\section{§ 0. Introduction}

The celebrated Ito formula for the $n$-dimensional Brownian motion $X_{t}$ and for $u \in C^{2}\left(\boldsymbol{R}^{n}\right)$ runs as follows:

$$
u\left(X_{t}\right)-u\left(X_{0}\right)=\sum_{i=1} \int_{0}^{t} \frac{\partial u}{\partial x_{i}}\left(X_{s}\right) d B_{s}+\int_{0}^{t} \frac{1}{2} \Delta u\left(X_{s}\right) d s, \quad B_{t}=X_{t}-X_{0} .
$$

In $\S 6$ of this paper we extend this to the case where $u$ is any element of the Sobolev space $H^{1}\left(\boldsymbol{R}^{n}\right)$ and accordingly $\Delta u$ is a tempered distribution which is not even a signed measure in general. As a consequence the second term of the right hand side of (0.1) may not be of bounded variation in $t$.

Successful attempts have been made to generalize (0.1) to more general processes $X_{t}$ than the Brownian motion (the formula due to KunitaWatanabe [6] and so on) or to more general functions $u$ than the $C^{2}$ functions (Tanaka's formula [9] and so on). They can be regarded as. specific realizations of semi-martingale decomposition [10]:

Semi-martingale $=$ martingale + process of bounded variation.

However, since the square integrable martingale of zero quadratic variation is identically zero, it seems to be natural and more general to conceive (0.1) as a decomposition into the sum

(0.2) martingale + process of zero quadratic variation.

In this paper we formulate a decomposition of the type (0.2) in an analytical way be making full use of the structure of the general symmetric Markov process. More specifically we introduce at the beginning of $\S 1$ the notion of the energy $e(A)$ of a (not necessary positive) additive functional $A$. We then establish in $\S 3$ a unique decomposition

Received July 20, 1978. 


$$
\tilde{u}\left(X_{t}\right)-\tilde{u}\left(X_{0}\right)=M_{t}^{[u]}+N_{t}^{[u]}, \quad M^{[u]} \in \ddot{\mathscr{M}}, \quad N^{[u]} \in \mathscr{N}_{\mathrm{c}}
$$

for any function $u$ in the associated Dirichlet space. Here $\mathscr{M}$ denotes the family of martingale additive functionals (MAF) of finite energy and $\mathscr{N}_{c}$ is the family of continuous additive functionals of zero energy.

The functional $N^{[u]}$ is characterized in $\S 4$ in a certain way by means of the element $T$ in the dual space $\mathscr{F}^{*}$ corresponding to $u$. In particular it is shown that $N_{t}^{[u]}$ is of bounded variation in $t$ if and only if the associated $T$ is expressed as a difference of smooth measures. The notion of the stochastic integral $f \cdot M \in \mathscr{M}$ based on the MAF $M$ is introduced in $\S 5$ with a certain relaxation of the integrability condition on $f$ imposed by Motoo-Watanabe [11]. These general theorems are applied in $\S 6$ to the Brownian motion, yielding the generalization of Ito's formula mentioned above together with a representation theorem of the space $\mathscr{M}$ by stochastic integrals.

This paper is actually a continuation of [4], so that before we come to our main topics, we shall give a quick review of what have been discussed there. The same notions and notations will appear without detailed interpretation. Now we start with a locally compact separable Hausdorff space $X$, an everywhere dense positive Radon measure $m$ on $X$ and an $m$-symmetric Hunt process $M=\left\{\Omega, X_{t}, \zeta, P_{x}\right\}$ on $X$. The associated Dirichlet form on $L^{2}(X ; m)$ is assumed to be regular. We put $\mathscr{F}$ $=\mathscr{D}[\mathscr{E}]$ and call this the Dirichlet space (relative to $L^{2}(X ; m)$ ). By an additive functional of $M$, we mean an ordinary additive functional with respect to the Hunt process $\left.M\right|_{X-B}, B$ being some proper exceptional set. Two additive functionals $A$ and $B$ are identified if $\forall t>0, P_{x}\left(A_{t}=B_{t}\right)=1$ q.e. $x \in X$. The effects of such relaxation of the definition of $A F$ are apparent in the previous paper and in the present one as well.

We also recall that the previous paper [4] has established a one-toone correspondence between the family $A_{c}^{+}$of positive continuous additive functionals (PCAF) and the family $S$ of smooth measures, the correspondence being specified by

$$
\lim _{t \downarrow 0} \frac{1}{t} E_{h \cdot m}\left((f \cdot A)_{t}\right)=\langle f \mu, h\rangle, \quad A \in A_{c}^{+}, \quad \mu \in S,
$$

for any $\gamma$-excessive function $h(\gamma \geqq 0)$ and any non-negative Borel $f$. 


\section{§1. Additive functionals of finite energy}

For any additive functional $A_{t}$ of the process $M$, we set

$$
e(A)=\lim _{t \downarrow 0} \frac{1}{2 t} E_{m}\left(A_{t}^{2}\right)
$$

when the limit exists. $e(A)$ is called the energy of $A$. Here are three important classes of AF's of finite energy:

\section{(I) AF's generated by functions}

Suppose that a function $u$ on $X$ possesses a version $\tilde{u}(u=\tilde{u}$ m-a.e.) such that $\tilde{u}$ is finely continuous q.e. and finite q.e. Then

$$
A_{t}^{[u]}=\tilde{u}\left(X_{t}\right)-\tilde{u}\left(X_{0}\right), \quad t \geqq 0
$$

defines an $\mathrm{AF}$ in our sense and indeed a unique equivalence class independent of the choice of the version $u$ ([3]).

When $u \in \mathscr{F}, A^{[u]}$ is well defined because we may take as $\tilde{u}$ a quasicontinuous version of $u$. Moreover $A_{t}^{[u]}$ is of finite energy and

$$
e\left(A^{[u]}\right)=\mathscr{E}^{\mathrm{res}}(u, u), \quad u \in \mathscr{F},
$$

by virtue of the formula (7.6) of the Appendices. Here $\mathscr{E}^{\text {res }}$ denotes the resurrected Dirichlet form defined by (7.5). $\mathscr{E}^{\text {res }}$ equals $\mathscr{E}$ if and only if there is no killing inside $X$ (Corollary to Proposition 7.1). When the process $M$ is transient, the relation (1.3) extends to all functions $u$ belonging to the extended Dirichlet space $\mathscr{F}_{e}$ (see Appendices).

\section{(II) Martingale additive functionals of finite energy}

Consider the family

$\mathscr{M}=\left\{M: M\right.$ is an $A F$ such that for each $t>0 E_{x}\left(M_{t}^{2}\right)<\infty$ and $E_{x}\left(M_{t}\right)=$ 0 q.e. $x \in X\}$.

Since $E_{m}\left(M_{t}^{2}\right)$ is subadditive in $t, e(M)$ is well defined and

$$
e(M)=\sup _{t>0} \frac{1}{2 t} E_{m}\left(M_{t}^{2}\right) \quad(\leqq+\infty)
$$

for any $M \in \mathscr{M}$. We set

$$
\ddot{\mathscr{U}}=\{M \in \mathscr{M}: e(M)<\infty\} .
$$


An AF $M$ belongs to the family $\mathscr{M}$ if and only if $M$ is a square integrable martingale $\mathrm{AF}$ in the ordinary sense with respect to the Hunt process $\left.\boldsymbol{M}\right|_{X-B}$, where $B$ is some proper exceptional set depending on $M$ in general. Hence the argument of P. A. Meyer [10; III. Théorème 3] applies and there exists for each $M \in \mathscr{M}$ a unique $\mathrm{AF}\langle M\rangle \in A_{c}^{+}$such that for any $t>0$

$$
E_{x}\left(\langle M\rangle_{t}\right)=E_{x}\left(M_{t}^{2}\right) \quad \text { q.e. } x \in X \text {. }
$$

We call $\langle M\rangle$ the quadratic variation of $M \in \mathscr{M}$. The smooth measure $\mu_{\langle M\rangle}$ corresponding to $\langle M\rangle$ by $(0.3)$ is called the energy measure of $\boldsymbol{M}$. From (1.1) and (1.6) we see that the energy of $M$ is just half of the total mass of its energy measure:

$$
e(M)=\frac{1}{2} \mu_{\langle M\rangle}(X) \quad M \in \mathscr{M} .
$$

\section{(III) CAF's of zero energy}

Let us put

$$
\begin{aligned}
\mathscr{N}_{c}= & \left\{N: N \text { is a continuous } A F \text { such that } e(N)=0 \text { and } E_{x}\left(\left|N_{t}\right|\right)\right. \text { is } \\
& \text { finite q.e. for each } t>0\} .
\end{aligned}
$$

The quadratic variation of $N \in \mathscr{N}_{c}$ vanishes in the following sense:

$$
\sum_{k=1}^{n T}\left(N_{(k+1) / n}-N_{k / n}\right)^{2} \rightarrow 0, \quad n \rightarrow \infty, \text { in } L^{1}\left(P_{m}\right),
$$

because the expectation of the left hand side equals

$$
\sum_{k=1}^{n T} E_{m}\left(E_{X_{k / n}}\left(N_{1 / n}^{2}\right)\right) \leqq n T \cdot E_{m}\left(N_{1 / n}^{2}\right) \rightarrow 0, \quad n \rightarrow \infty .
$$

An example of $N \in \mathscr{N}_{c}$ is given by

$$
N_{t}=\int_{0}^{t} f\left(X_{s}\right) d s
$$

for any Borel function $f \in L^{2}(X ; m)$. Then $E_{x}\left(\int_{0}^{t}\left|f\left(X_{s}\right)\right| d s\right) \leqq e^{t} R_{1}|f|(x)$ is finite for q.e. $x \in X$ and $N$ is a CAF. Furthermore

$$
\begin{aligned}
E_{m}\left(N_{t}^{2}\right) & =2 E_{m}\left(\int_{0}^{t} f\left(X_{s}\right) \int_{s}^{t} f\left(X_{v}\right) d v d s\right)=2 E_{m}\left(\int_{0}^{t} \int_{0}^{t-s} f\left(X_{s}\right) p_{v} f\left(X_{s}\right) d v d s\right) \\
& =2 \int_{0}^{t} \int_{0}^{t-s}\left(p_{s} 1, f \cdot p_{v} f\right) d v d s \text {. Hence }
\end{aligned}
$$




$$
\frac{1}{2 t} E_{m}\left(N_{t}^{2}\right)=\frac{1}{t} \int_{0}^{t}\left(p_{t-s} 1, f \cdot S_{s} f\right) d s \quad \text { with } \quad S_{s} f(x)=\int_{0}^{s} p_{v} f(x) d v
$$

Since the right hand side of (1.11) is dominated by $\frac{1}{t} \int_{0}^{t} s d s \cdot(f, f), N$ of (1.10) is of zero energy, namely $N \in \mathscr{N}_{c}$.

In this paper we are particularly interested in the sum of the classes (II) and (III):

$$
\mathscr{A}=\ddot{\mathscr{M}} \oplus \mathscr{N}_{c}
$$

namely, $\mathscr{A}$ consists of AF's $A$ such that

$$
A_{t}=M_{t}+N_{t}, \quad M \in \check{\mathscr{M}}, N \in \mathscr{N}_{c} .
$$

Evidently $\mathscr{A}$ is a linear space of AF's of finite energy. Moreover the expression (1.13) of $A \in \mathscr{A}$ is unique because

$$
\ddot{\mathscr{M}} \cap \mathscr{N}_{c}=\{0\}
$$

where 0 denotes the additive functional identically zero. In fact, if $A \in \mathscr{M}$ is of zero energy, then $\mu_{\langle A\rangle}$ vanishes by (1.7) and so does $\langle A\rangle$. Hence $A=0$ by (1.6).

We define the mutual energy of $A, B \in \mathscr{A}$ by

$$
e(A, B)=\lim _{t ! 0} \frac{1}{2 t} E_{m}\left(A_{t} B_{t}\right) \text {. }
$$

We know by Schwarz inequality that $e(A, B)=0$ when either $A$ or $B$ is in $\mathscr{N}_{c}$. Therefore

$$
e(A)=e(M) \quad \text { if } A=M+N, M \in \mathscr{M}, N \in \mathscr{N}_{c} .
$$

The main purpose of this paper is to show that any $\operatorname{AF} A^{[u]}(u \in \mathscr{F})$ in the first class (I) actually belongs to $\mathscr{A}$, which is enough to get a unique decomposition of $A^{[u]}$ indicated in Introduction. We first prove that the space $\mathscr{M}$ is a Hilbert space with inner product (1.15).

\section{§. Completeness of the space $(\ddot{\mathscr{M}}, e)$}

TheOREm 1. $\mathscr{M}$ is a real Hilbert space with inner product e. More specifically, if $M^{(n)} \in \mathscr{M}$ constitutes a Cauchy sequence with respect to $e$, then there exist a unique $M \in \mathscr{M}$ and a subsequence $n_{k} \rightarrow \infty$ such that

$$
\lim _{n \rightarrow \infty} e\left(M^{(n)}-M\right)=0, \text { and for q.e. } x \in X \text {, }
$$


$P_{x}\left(M_{t}^{\left(n_{k}\right)}\right.$ converges uniformly in any finite interval of $\left.t\right)=1$.

The proof is based on the next lemma.

Lemma 1. Consider $A \in A_{c}^{+}$and the associated smooth measure $\mu_{A}$. Then for any $\nu \in S_{00}$ and $t>0$

$$
E_{\nu}\left(A_{t}\right) \leqq(1+t)\left\|U_{1} \nu\right\|_{\infty} \cdot \mu_{A}(X) \quad(\leqq \infty) .
$$

Proof. Assume first that $\mu=\mu_{A}$ is of finite energy integral: $\mu \in \boldsymbol{S}_{0}$. By setting $c_{t}(x)=E_{x}\left(A_{t}\right), x \in X$, we claim that

$$
c_{t} \in \mathscr{F} \quad \text { and } \quad \mathscr{E}\left(c_{t}, v\right)=\left\langle\mu, \tilde{v}-p_{t} \tilde{v}\right\rangle, \forall v \in \mathscr{F} .
$$

By Proposition 1 of [4], the potential $u_{A}^{1}(x)$ of $A$ is a quasicontinuous version of the potential $U_{1} \mu$ of $\mu$. Hence $\left\langle\mu, c_{t}\right\rangle \leqq e^{t}\left\langle\mu, u_{A}^{1}\right\rangle=e^{t} \mathscr{E}_{1}(\mu)<\infty$. On the other hand, $\frac{1}{s}\left(c_{t}, c_{t}-p_{s} c_{t}\right)=\frac{1}{s}\left(c_{t}, c_{s}-p_{t} c_{s}\right)=\frac{1}{s}\left(c_{t}-p_{t} c_{t}, c_{s}\right)$ which is, by Lemma 9 of [4], equal to $\frac{1}{s} \int_{0}^{s}\left\langle\mu, p_{u}\left(c_{t}-p_{t} c_{t}\right)\right\rangle d u=\frac{1}{s} \int_{0}^{s}\langle\mu$, $\left.2 c_{t+u}-c_{u}-c_{2 t+u}\right\rangle d u$. Therefore we get $\lim _{s ! 0} \frac{1}{s}\left(c_{t}, c_{t}-p_{s} c_{t}\right)=\left\langle\mu, 2 c_{t}-c_{2 t}\right\rangle$ $=\left\langle\mu, c_{t}-p_{t} c_{t}\right\rangle\left\langle\infty\right.$, proving that $c_{t} \in \mathscr{F}$ and $\mathscr{E}\left(c_{t}, c_{t}\right)=\left\langle\mu, c_{t}-p_{t} c_{t}\right\rangle$. In the same way we have the relation of (2.2).

(2.2) leads us to, for $\mu \in S_{0}$ and $\nu \in S_{00}, E_{\nu}\left(A_{t}\right)=\left\langle\nu, c_{t}\right\rangle=\mathscr{E}_{1}\left(U_{1} \nu, c_{t}\right)$ $=\left\langle\mu, U_{1} \nu-p_{t}\left(U_{1} \nu\right)\right\rangle+\left(c_{t}, U_{1} \nu\right) \leqq\left\|U_{1} \nu\right\|_{\infty}\left(\mu(X)+\int_{X} c_{t}(x) m(d x)\right)$, which proves the inequality (2.1) by noting that $\sup _{t>0} \frac{1}{t} \int_{X} c_{t}(x) m(d x)=\lim _{t \downarrow 0} \frac{1}{t} E_{m}\left(A_{t}\right)$ $=\mu(X)$.

When $\mu=\mu_{A}$ is a general smooth measure, we may consider the increasing sequence $\left\{F_{n}\right\}$ of closed sets of Lemma 3 of [4]. Since $I_{F_{n}} \cdot A$ is related to $I_{F_{n}} \cdot \mu \in S_{0}$, we have

$$
E_{\nu}\left(\left(I_{F_{n}} \cdot A\right)_{t}\right) \leqq(1+t)\left\|U_{1} \nu\right\|_{\infty} \cdot \mu\left(F_{n}\right) .
$$

By letting $n$ tend to infinity, we arrive at (2.1).

q.e.d.

Proof of Theorem 1. Lemma 1 together with (1.6) and (1.7) implies for any $M \in \mathscr{M}$

$$
E_{\nu}\left(M_{t}^{2}\right) \leqq 2(1+t)\left\|U_{1} \nu\right\|_{\infty} e(M), \quad \nu \in S_{00} .
$$

$\left(M_{t}, P_{\nu}\right)_{t \geqq 0}$ is then a square integrable martingale and we have 


$$
P_{\nu}\left(\sup _{0 \leqq s \leqq T}\left|M_{s}\right|>\alpha\right) \frac{2}{\alpha^{2}}(1+T)\left\|U_{1} \nu\right\|_{\infty} e(M)
$$

on account of Doob's inequality.

Assume that $M^{(n)} \in \mathscr{M}$ constitutes an $e$-Cauchy sequence and select a subsequence $n_{k} \rightarrow \infty$ such that $e\left(M^{\left(n_{k+1}\right)}-M^{\left(n_{k}\right)}\right) \leqq \frac{1}{2^{3 k}}$. By (2.4)

$$
P_{\nu}\left(\sup _{0 \leqq s \leqq T}\left|M_{s}^{\left(n_{k+1}\right)}-M_{s}^{\left(n_{k}\right)}\right|>\frac{1}{2^{k}}\right) \leqq \frac{2(1+T)\left\|U_{1^{\nu}}\right\|_{\infty}}{2^{k}} .
$$

Applying the Borel-Cantelli lemma to this inequality, we see

$$
P_{\nu}\left(\Lambda_{0}\right)=1, \quad \nu \in S_{00}
$$

where $\Lambda_{0}=\left\{\omega \in \Omega ; M_{s}^{\left(n_{k}\right)}\right.$ converges uniformly in $s$ on each finite interval $\}$.

In view of Lemma 1 of [4], (2.5) implies $P_{x}\left(\Lambda_{0}\right)=1$ q.e. $x \in X$. Denote by $\Gamma_{k}$ a defining set of $M^{\left(n_{k}\right)}$ and put $\Lambda_{1}=\bigcap_{k} \Gamma_{k}$. We further put for $\omega$ $\in A_{0} \cap A_{1} M_{s}(\omega)=\lim _{n_{k} \rightarrow \infty} M_{s}^{\left(n_{k}\right)}(\omega), s \geqq 0 . \quad M_{s}$ is then an AF with defining set $\Lambda_{0} \cap \Lambda_{1}$. Since $M_{t}^{(n)}$ is $L^{2}\left(P_{\nu}\right)$ convergent to $M_{t}$ by virtue of (2.3), we see $E_{\nu}\left(M_{t}^{2}\right)<\infty$ and $E_{\nu}\left(M_{t}\right)=0$ for any $\nu \in S_{00}$. It is easy to conclude from this with the help of Lemma 1 of [4] that $E_{x}\left(M_{t}^{2}\right)<\infty$ and $E_{x}\left(M_{t}\right)=0$ q.e. $x \in X$, that is, $M \in \mathscr{M}$.

For any $\varepsilon>0$, choose $N$ such that $e\left(M^{(n)}-M^{(m)}\right)<\varepsilon, n, m>N$. We have $\frac{1}{2 t} E_{m}\left(\left(M_{t}^{(n)}-M_{t}\right)^{2}\right) \leqq \varepsilon$ by Fatou's lemma and (1.4). Then $e\left(M^{(n)}-M\right)$ $\leqq \varepsilon$. Hence $M \in \mathscr{M}$ and $M^{(n)}$ is e-convergent to $M$. q.e.d.

§3. Decomposition of $A_{t}^{[u]}=\tilde{u}\left(X_{t}\right)-\tilde{u}\left(X_{0}\right), u \in \mathscr{F}$

Theorem 2. For any $u \in \mathscr{F}$, the $A F A^{[u]}$ belongs to the class $\mathscr{A}$ $\mathscr{M} \oplus \mathscr{N}_{c}$ and so that $A^{[u]}$ can be expressed uniquely as

$$
A^{[u]}=M^{[u]}+N^{[u]}, \quad M^{[u]} \in \mathscr{M}, \quad N^{[u]} \in \mathscr{N}_{c} .
$$

Moreover it holds that

$$
e\left(M^{[u]}\right)=\mathscr{E}^{\mathrm{res}}(u, u) .
$$

In transient case, the above statements extend to any $u \in \mathscr{F}_{e}$ (see Appendices for notions).

Proof. We already know in $\S 1$ the uniqueness of the decomposition 
(3.1) and hence it suffices to show the existence of such $M^{[u]}$ and $N^{[u]}$.

We start with a special case that $u$ is in the range of the resolvent: $u=R_{1} f, R_{\alpha}$ being the resolvent of the process $M$ and $f$ being a Borel function in $L^{2}(X ; m)$. In this case (3.1) is reduced to the usual semimartingale decomposition, namely, we may put

$$
\left\{\begin{array}{l}
N_{t}^{[u]}=\int_{0}^{t}\left(u\left(X_{s}\right)-f\left(X_{s}\right)\right) d s \\
M_{t}^{[u]}=u\left(X_{t}\right)-u\left(X_{0}\right)-N_{t}^{[u]}, \quad t \geqq 0
\end{array}\right.
$$

As we saw in $\S 1$ (III), $N^{[u]}$ of (3.3) belongs to $\mathscr{N}_{c}$. Since $u$ is an element of $\mathscr{F}, A_{t}^{[u]}=u\left(X_{t}\right)-u\left(X_{0}\right)$ is of finite energy and $M_{t}^{[u]}$ of (3.3) satisfies the relation '(3.2) in view of (1.3). Since $\left\langle\nu, R_{1} f^{2}\right\rangle \leqq\left\|U_{1} \nu\right\|_{\infty} \cdot(f, f)<\infty$, $\forall \nu \in S_{o o}$, we can see that $M^{[u]} \in \mathscr{M}$ and hence $M^{[u]} \in \mathscr{M}$.

Next take any Borel function $u \in \mathscr{F}$ and define the approximating functions $u_{n}$ by

$$
u_{n}=n R_{n+1} u=R_{1} f_{n} \quad \text { with } f_{n}=n\left(u-n R_{n+1} u\right) .
$$

By the uniqueness of the decomposition (3.1) for $u_{n}$ 's, we have then $M^{\left[u_{n}\right]}-M^{\left[u_{m}\right]}=M^{\left[u_{n}-u_{m}\right]}$ and

$$
e\left(M^{\left[u_{n}\right]}-M^{\left[u_{m}\right]}\right)=\mathscr{E}^{\text {res }}\left(u_{n}-u_{m}, u_{n}-u_{m}\right) .
$$

Since $u_{n} \in \mathscr{F}$ are $\mathscr{E}_{1}$-convergent to $u$ and $\mathscr{E}_{1}(v, v)$ dominates $\mathscr{E}^{\text {res }}(v, v)$ for any $v \in \mathscr{F}$, we conclude that $\left\{M^{\left[u_{n}\right]}\right\}$ is an $e$-Cauchy sequence in the space $\ddot{\mathscr{M}}$.

By virtue of Theorem 1, the formula

$$
\begin{cases}M^{[u]}=\lim _{n \rightarrow \infty} M^{\left[u_{n}\right]} & \text { in }(\mathscr{\mathscr { M }}, e) \\ N^{[u]}=A^{[u]}-M^{[u]}, & u \in \mathscr{F}\end{cases}
$$

makes sense and

$$
M^{[u]} \in \ddot{\mathscr{M}}, \quad e\left(M^{[u]}\right)=\mathscr{E}^{\mathrm{res}}(u, u), \quad u \in \mathscr{F} .
$$

It only remains to show that $N^{[u]}$ of (3.6) belongs to the space $\mathscr{N}_{c}$. Notice that a subsequence $n_{k}$ exists and

$$
\begin{aligned}
& P_{x}\left(N_{t}^{\left[u_{n k}\right]} \text { converges to } N_{t}^{[u]} \text { uniformly on any finite interval of } t\right) \\
& =1 \text { q.e. } x \in X,
\end{aligned}
$$

because the same statements for $A^{\left[u_{n}\right]}$ and $M^{\left[u_{n}\right]}$ hold on account of 
Theorem 1 and [4: Lemma 6] respectively. From this and (3.3), we know that $N^{[u]}$ is a CAF.

On the other hand we have from (3.6) $N_{t}^{[u]}=A_{t}^{\left[u-u_{n}\right]}-\left(M_{t}^{[u]}-M_{t}^{\left[u_{n}\right]}\right)$ $+N_{t}^{\left[u_{n}\right]}$ and consequently

$$
\varlimsup_{t \downarrow 0} \frac{1}{2 t} E_{m}\left(\left(N_{t}^{[u]}\right)^{2}\right) \leqq 3 e\left(A^{\left[u-u_{n}\right]}\right)+3 e\left(M^{\left[u-u_{n}\right]}-M^{\left[u_{n}\right]}\right) .
$$

Owing to (1.3), (3.5) and (3.6), the right hand side equals $6 \mathscr{E}^{\text {res }}\left(u-u_{n}\right.$, $\left.u-u_{n}\right)$, which can be made arbitrarily small with large $n$. Therefore $e\left(N^{[u]}\right)=0$ and $N^{[u]} \in \mathscr{N}_{c}$.

When the process is transient, we may consider the extended Dirichlet space $\left(\mathscr{F}_{e}, \mathscr{E}\right)$ according to Appendices. Then exactly in the same way as above, we can extend the decomposition (3.1) and the relation (3.2) to any $u \in \mathscr{F}_{e}$ by making use of an approximating sequence $u_{n} \in \mathscr{F}$ which is $\mathscr{E}$-convergent to $u \in \mathscr{F}_{e}$. q.e.d.

Corollary 1. The following linearity and continuity hold:

(i) $\quad M^{[a u+b v]}=a M^{[u]}+b M^{[v]}, N^{[a u+b v]}=a N^{[u]}+b N^{[v]}, a, b \in R^{1}, u, v \in \mathscr{F}(u$, $v \in \mathscr{F}_{e}$ in transient case).

(ii) $u_{n}, u \in \mathscr{F}\left(u_{n}, u \in \mathscr{F}_{e}\right.$ in transient case), $\mathscr{E}\left(u_{n}-u, u_{n}-u\right) \rightarrow 0, n \rightarrow \infty$ $\Rightarrow{ }^{\exists} n_{k}$ a subsequence of $n: \lim _{n_{k} \rightarrow \infty} M_{t}^{\left[u_{n_{k}}\right]}=M_{t}^{[u]}, \lim _{n_{k} \rightarrow \infty} N_{t}^{\left[u_{n_{k}}\right]}=N_{t}^{[u]}$ uniformly on each finite interval of $t, P_{x}$-a.s. for q.e. $x \in X$.

In fact, the relation (i) follows from the uniqueness of the decomposition (3.1). The equality (3.2) means that the linear transform

$$
\Phi: u \backsim M^{[u]}
$$

is continuous from the Dirichlet space $(\mathscr{F}, \mathscr{E})$ (the extended Dirichlet space $\left(\mathscr{F}_{e}, \mathscr{E}\right)$ in transient case) into the space $(\mathscr{M}, e)$. Combining this with Theorem 1 and [4; Lemma 6], we conclude the continuity statement (ii).

(3.2) also implies the following embedding statement telling us that the structure of the Dirichlet space may be studied entirely within the framework of the space $\mathscr{M}$ of martingales of finite energy.

Corollary 2. Suppose that the process $M$ is transient and that there is no killing inside $X$. Then the transformation $\Phi$ of (3.9) is isometry and the extended Dirichlet space $\left(\mathscr{F}_{e}, \mathscr{E}\right)$ can be identified by $\Phi$ with a closed subspace of $(\mathscr{M}, e)$. 


\section{$\S 4$. Characterizations of $N^{[u]}$}

We saw in $\S 1$ (III) that any $\mathrm{AF}$ in $\mathscr{N}_{c}$ is of zero quadratic variation in a certain sense. Nevertheless the class $\mathscr{N}_{c}$ contains in general a lot of CAF's which are not of bounded variation. In this section we make the situation clear by giving some characterizations of the CAF $N^{[u]}$ constructed in the preceding section for $u \in \mathscr{F}$ ( $u \in \mathscr{F}_{e}$ in transient case). See $\S 6$ for some related examples.

Theorem 3. Following conditions are equivalent for an $A F A$ and for a function $u \in \mathscr{F}\left(u \in \mathscr{F}_{e}\right.$ in transient case):

(i) $A=N^{[u]}$

(ii) $A \in \mathscr{N}_{c}$ and, for each $t>0$,

$$
E_{x}\left(A_{t}\right)=p_{t} u(x)-u(x), \text { q.e. } x \in X .
$$

(iii) $A \in \mathcal{N}_{c}, \lim _{t \downarrow 0} E_{x}\left(A_{t}\right)=0$ q.e. and

$$
\lim _{t \downarrow 0} \frac{1}{t} E_{\imath \bullet m}\left(A_{t}\right)=-\mathscr{E}(u, v), \quad \forall v \in \mathscr{F} .
$$

Proof. The implication (i) $\Rightarrow$ (ii) $\Rightarrow$ (iii) is clear. Assume that an AF $A$ satisfies the condition (iii). Let us put $c_{t}(x)=E_{x}\left(A_{t}\right)$, then we see from $A \in \mathscr{N}_{c}$ and the relation

$$
c_{t+s}(x)=c_{t}(x)+p_{t} c_{s}(x) \text { q.e. } \quad \text { for each } t, s>0,
$$

that $c_{t} \in L^{2}(X ; m), t>0$.

By (4.2) and (4.3), we have for any $v \in \mathscr{F}, t>0$, and $T>0$,

$$
\begin{aligned}
& \left(v-p_{T} v, c_{t}\right)=\lim _{s \downarrow 0} \frac{1}{s}\left(S_{T} v,-p_{s} S_{T} v, c_{t}\right)=\lim _{s \downarrow 0} \frac{1}{s}\left(S_{T} v-p_{t} S_{T} v, c_{s}\right) \\
& \quad=-\mathscr{E}\left(S_{T} v-p_{t} S_{T} v, u\right)=-\mathscr{E}\left(S_{T} v, u-p_{t} u\right)=-\left(v-p_{T} v, u-p_{t} u\right) .
\end{aligned}
$$

Hence, $l_{t}=\left(v, c_{t}+u-p_{t} u\right)$ is linear in $t ; l_{t}=l_{t+T}-l_{T}$. Since $\lim _{s \downarrow 0} \frac{1}{t} l_{T}=0$ by (4.2), $l_{t}=0$ and, consequently,

$$
c_{t}=p_{t} u-u \quad m \text {-a.e. }
$$

(4.4) particularly means that $c_{t} \in \mathscr{F}\left(c_{t} \in \mathscr{F}_{e}\right.$ in transient case). Moreover $c_{t}(x)$ is a q.e. limit of a quasi-continuous function $p_{s} c_{t-s}(x)$ in view of (4.3) and a condition in (iii). Hence $c_{t}$ is quasi-continuous and (4.1) follows from (4.4). The implication (iii) $\Rightarrow$ (ii) is proven. 
Finally we assume condition (ii) for $A$. In order to derive (i), we put $B_{t}=N_{t}^{[u]}-A_{t}=\tilde{u}\left(X_{t}\right)-\tilde{u}\left(X_{0}\right)-M_{t}^{[u]}-A_{t}, t>0, q_{t}(x)=E_{x}\left(B_{t}^{2}\right)$ and claim that

$$
q_{t}(x)=0 \quad \text { q.e. } \quad \text { for each } t>0 .
$$

Clearly $B_{t}$ is a CAF of zero energy and $E_{x}\left(B_{t}\right)=0$ on account of the assumption (ii). But then $\int_{X} q_{t}(x) m(d x)=0$ because the left hand side is subadditive in $t$. We thus have $q_{t}(x)=0 \mathrm{~m}$-a.e., from which we can conclude $p_{s} q_{t}=0$ q.e. for each $s>0$. By using Fatou's lemma $q_{t}(x)$ $=E_{x}\left(\varliminf_{n \rightarrow \infty}\left(B_{t+(1 / n)}-B_{1 / n}\right)^{2}\right) \leqq \varliminf_{n \rightarrow \infty} p_{1 / n} q_{t}(x)=0$ q.e., getting (4.5). $\quad$ q.e.d.

An $\mathrm{AF} A$ is said to be of bounded variation if $A_{t}(\omega)$ is of bounded variation in $t$ on each compact subinterval of $[0, \zeta(\omega))$ for every fixed $\omega$ in a defining set of $A$. A CAF $A$ is of bounded variation if and only if $A$ can be expressed as a difference of two PCAF's:

$$
A_{t}(\omega)=A_{t}^{(1)}(\omega)-A_{t}^{(2)}(\omega), \quad A^{(1)}, A^{(2)} \in A_{c}^{+}
$$

If an $\mathrm{AF} A$ is continuous and of bounded variation, then its total variation $\{A\}\left(=\int_{0}^{t}\left|d A_{s}\right|\right)$ is a PCAF ([10, III, Proposition 1]) and an expression (4.6) is provided by $A_{t}^{(1)}=\{A\}_{t}, A^{(2)}=\{A\}_{t}-A_{t}$ for instance.

In [4], we have introduced the space $S_{0}$ of positive Radon measures on $X$. For definiteness we say each measure $\nu \in S_{0}$ to be of finite 1-order energy integral. In transient case, the same role is played by a measure $\nu$ of finite 0-order energy integral which is defined as a positive Radon measure satisfying

$$
\int_{X}|v(x)| \nu(d x) \leqq C\|v\|_{\delta}, \quad v \in \mathscr{F} \cap C_{0}(X) .
$$

Let us say that a signed Radon measure $\nu$ on $X$ is of bounded 1-order (resp. 0-order) energy integral if so is the total variation $|\nu|$. This is the case if and only if $\nu$ can be expressed as $\nu=\nu^{(1)}-\nu^{(2)}, \nu^{(1)}$ and $\nu^{(2)}$ being positive Radon measures of the same type. We can then define the $\alpha$ potential of $\nu$ by $U_{\alpha} \nu=U_{\alpha} \nu^{(1)}-U_{\alpha} \nu^{(2)}$ (resp. 0-potential of $\nu$ by $U_{\nu}=U_{\nu}^{(1)}$ $\left.-U \nu^{(2)}\right)$ which does not depend on the choice of $\nu^{(1)}$ and $\nu^{(2)}$. Moreover the quasi-continuous version $\tilde{v}$ of any $v \in \mathscr{F}$ (resp. $v \in \mathscr{F}_{e}$ ) is integrable with respect to the total variation $|\nu|$ and it holds that 


$$
\begin{array}{ll}
\mathscr{E}_{\alpha}\left(U_{\alpha} \nu, v\right)=\langle\nu, \tilde{v}\rangle & \forall v \in \mathscr{F} \\
\mathscr{E}(U \nu, v)=\langle\nu, \tilde{v}\rangle & \forall v \in \mathscr{F}_{e}
\end{array}
$$

respectively.

Given a signed Radon measure $\nu$ of finite 1-order (resp. 0-order in transient case) energy integral, we express it as $\nu=\nu^{(1)}-\nu^{(2)}$ in the above way and put

$$
A=A^{(1)}-A^{(2)}
$$

where $A^{(1)}$ and $A^{(2)}$ are PCAF corresponding to $\nu^{(1)}$ and $\nu^{(2)}$ respectively. $A$ of (4.9) is a CAF of bounded variation and does not depend on the choice of $\nu^{(1)}$ and $\nu^{(2)}$.

LemMa 2. For any signed Radon measure as above, it holds that

$$
N_{t}^{\left[U_{1} \nu\right]}=\int_{0}^{t} \widetilde{U_{1} \nu}\left(X_{s}\right) d s-A_{t}, \quad t \geqq 0,
$$

and in transient case that

$$
N^{[U \nu]}=-A,
$$

where $A$ is the CAF of bounded variation associated with $\nu$ by (4.9).

Proof. In view of the linearity (Corollary 1 to Theorem 2), we may assume that $\nu$ is non-negaive. Let us first prove (4.10).

Now take $\nu \in S_{0}$ and a quasi-continuous version $u$ of $U_{1} \nu$. By virtue of (3.6) and (3.8), we then have

$$
N_{t}^{[u]}=\lim _{n \rightarrow \infty} \int_{0}^{t}\left(n R_{n+1} u\left(X_{s}\right)-f_{n}\left(X_{s}\right)\right) d s
$$

where $f_{n}=n\left(u-n R_{n+1} u\right)$ and the convergence in (4.12) is uniform in $t$ on each finite interval $P_{x}$-a.s. for q.e. $x \in X$. The equality (4.10) then follows immediately from (4.12) together with [4; Lemma 6] and the way of the construction of $A \in A_{c}^{+}$carried out in [4; Proposition 1].

In order to prove (4.11), observe that any positive Radon measure of finite 0-order energy integral is also an element of $\boldsymbol{S}_{0}$ and

$$
U_{\nu}=U_{1} \nu+R\left(\widetilde{U_{1}^{\top} \nu}\right)
$$


where $R$ denotes the 0 -order resolvent of the process $M$.

Put $f=\widetilde{U_{1} \nu}$, then, in view of Appendices, $R_{\alpha} f(\in \mathscr{F})$ converges as $\alpha \downarrow 0$ to $R f$ in $\left(\mathscr{F}_{e}, \mathscr{E}\right)$. Therefore, by the continuity again and by an obvious modification of [4; Lemma 6$]$, we see

$$
N_{t}^{[R f]}=\lim _{\alpha \downarrow 0} N_{t}^{[R \alpha f]}=\lim _{\alpha \downarrow 0} \int_{0}^{t}\left(\alpha R_{\alpha} f\left(X_{s}\right)-f\left(X_{s}\right)\right) d s=-\int_{0}^{t} f\left(X_{s}\right) d s .
$$

(4.11) follows from this and (4.13).

q.e.d.

Before formulating the next theorem, recall Lemma 3 of [4] (resp. its obvious modification in transient case): if a non-negative Borel measure $\nu$ is smooth, then there exists an increasing sequence $\left\{F_{k}\right\}$ of closed set satisfying

$$
P_{x}\left(\lim _{k \rightarrow \infty} \sigma_{X-F_{k}}<\zeta\right)=0 \quad \text { q.e. } \quad x \in X
$$

(4.15) $I_{F_{k}} \cdot \nu \in S_{0}$ (resp. $I_{F_{k}} \cdot \nu$ is of finite 0-order energy integral) for each $k$.

Let us call such $\left\{F_{k}\right\}$ a nest associated with $\nu \in S$.

Given two smooth measures $\nu^{(1)}$ and $\nu^{(2)}$, we can always choose a common nest $\left\{F_{k}\right\}$ associated with $\nu^{(1)}$ and $\nu^{(2)}$. We put then

$$
\nu_{k}=I_{F_{k}} \cdot \nu^{(1)}-I_{F^{\prime}} \cdot \nu^{(2)}, \quad k=1,2, \cdots,
$$

and call $\nu_{k}$ the restriction to $F_{k}$ of the difference $\nu^{(1)}-\nu^{(2)}$. Each $\nu_{k}$ is a signed Radon measure of finite 1-order energy integral (resp. of finite 0 -order energy integral in transient case). It we put

$$
\mathscr{F}_{k}=\left\{u \in \mathscr{F} ; \tilde{u}=0 \text { q.e. on } X-F_{k}\right\} \text {, }
$$

then on account of [4; Lemma 7] and (4.7),

$$
\mathscr{E}_{\alpha}\left(U_{\alpha} \nu_{k}-H_{\alpha, k}\left(\widetilde{U_{\alpha} \nu_{k}}\right), v\right)=\left\langle\nu_{k}, \tilde{v}\right\rangle, \forall v \in \mathscr{F}_{k},
$$

where $H_{\alpha, k} f(x)=E_{x}\left(e^{-\alpha \sigma k} u\left(X_{\sigma_{k}}\right)\right), \sigma_{k}=\sigma_{X-F k}$.

The same statement for $\alpha=0$ holds in transient case. Keeping these in mind we proceed to the proof of the next theorem.

THEOREM 4. Following two conditions are equivalent each other for $u \in \mathscr{F}\left(u \in \mathscr{F}_{e}\right.$ in transient case): 
(i) $N^{\prime u]}$ is a CAF of bounded variation.

(ii) there exist smooth measures $\nu^{(1)}$ and $\nu^{(2)}$ such that

$$
\mathscr{E}(u, v)=\left\langle\nu_{k}, \tilde{v}\right\rangle, \quad \forall v \in \mathscr{F}_{k},
$$

for every $k$. Here $\nu_{k}$ is the restriction to $F_{k}$ of the difference $\nu^{(1)}-\nu^{(2)},\left\{F_{k}\right\}$ being a common nest associated with $\nu^{(1)}$ and $\nu^{(2)} . \mathscr{F}_{k}$ is the space defined by (4.17).

Moreover in this case $N^{[u]}$ is characterized as the unique CAF A of bounded variation satisfying for every $k$

$$
\lim _{t \downarrow 0} \frac{1}{t} E_{v \cdot m}\left(A_{t}\right)=-\left\langle\nu_{k}, \tilde{v}\right\rangle, \quad \forall v \in \mathscr{F}_{k}
$$

Proof. We only give the proof for $u \in \mathscr{F}$.

Proof of (i) $\Rightarrow$ (ii): Suppose $N^{[u]} \in \mathscr{N}_{c}$ is of bounded variation, then $N^{[u]}$ $=-A^{(1)}+A^{(2)}$ for some $A^{(1)}$ and $A^{(2)} \in A_{c}^{+}$. Denote by $\nu^{(1)}$ and $\nu^{(2)}$ the smooth measures associated with $A^{(1)}$ and $A^{(2)}$ respectively. Then (4.20) holds for a function $v=h-H_{1, k} h\left(\in \mathscr{F}_{k}\right), h$ being any 1-excessive function in $\mathscr{F}$ in accordance with (0.3). Hence the relation (4.19) is valid for such type of functions in view of Theorem 3. For any $v \in \mathscr{F}_{k}$, we can choose a sequence $\left\{v_{n}\right\}$ of the above type which is $\mathscr{E}_{1}$-convergent to $v$. Since $\left|\nu_{k}\right|$ is of finite 1-energy integral, $v_{n}$ is $L^{1}\left(X ;\left|\nu_{k}\right|\right)$-convergent to $v$ as well. Thus (4.19) is established for any $v \in \mathscr{F}_{k}$ for each $k$.

Proof of (ii) $\Rightarrow$ (i): Assume that the relation (ii) holds. Let $A^{(1)}$ and $A^{(2)}$ be the PCAF associated with the smooth measures $\nu^{(1)}$ and $\nu^{(2)}$ respectively. We set $A=-A^{(1)}+A^{(2)}$ and claim that

$$
N^{[u]}=A \text {. }
$$

Fix $k$ and rewrite (4.19) as

$$
\mathscr{E}_{1}(u, v)=\left\langle\mu_{k}, \tilde{v}\right\rangle \quad \forall v \in \mathscr{F}_{k},
$$

where $\mu_{k}=u \cdot m+\nu_{k}$. Since the left hand side of (4.22) equals to $\mathscr{E}_{1}\left(u-H_{1, k} \tilde{u}, v\right)$ for any $v \in \mathscr{F}_{k}$, we get from (4.18) and (4.22)

$$
u-H_{1, k} \tilde{u}=U_{1} \mu_{k}-H_{1, k}\left(\widetilde{U_{1} \mu_{k}}\right) \text {. }
$$

Now the $\mathrm{CAF} I_{F_{k}} \cdot A=-I_{F_{k}} \cdot A^{(1)}+I_{F_{k}} \cdot A^{(2)}$ is associated with the 
measure $-\nu_{k}$. Hence $\int_{0}^{t} u\left(X_{s}\right) d s-\left(I_{F_{k}} \cdot A\right)_{t}$ is associated with $\mu_{k}$ and by Lemma 2 we arrive at the equality

$$
N_{t}^{\left[U_{1} \mu_{k}\right]}=\int_{0}^{t}\left\{\widetilde{U_{1} \mu_{k}}\left(X_{s}\right)-u\left(X_{s}\right)\right\} d s+\left(I_{F_{k}} \cdot A\right)_{t}, \quad t>0 .
$$

On the other hand, $H_{1, k}\left(\widetilde{U_{1} \mu_{k}}\right)$ is also a 1-potential of a measure $\pi_{k} \mu_{k} \in S_{0}$ supported by $\overline{X-F_{k}}$ ([4; Lemma 7]). Denote by $B$ the PCAF associated with $\pi_{k} \mu_{k}$. Then using Lemma 2 again

$$
N_{t}^{\left[H _ { 1 , k } \left(\widetilde{\left.\left.U_{1} \mu_{k}\right)\right]}\right.\right.}=\int_{0}^{t} H_{1, k}\left(\widetilde{U_{1} \mu_{k}}\right)\left(X_{s}\right) d s-B_{t}, \quad t \geqq 0 .
$$

(4.23), (4.24), (4.25) and Corollary to Theorem 2 imply

$$
N_{t}^{\left[u-H_{1, k} \tilde{u}\right]}=-\int_{0}^{t} H_{1, k} \tilde{u}\left(X_{s}\right) d s+\left(I_{F k} \cdot A\right)_{t}+B_{t}, \quad t>0 .
$$

Since $B_{t}=0,{ }^{\forall} t<\sigma_{k}$ we then conclude

$$
N_{t}^{\left[u-H_{1, k} \tilde{u}\right]}=-\int_{0}^{t} H_{1, k}\left(X_{s} \tilde{u}\right) d s+A_{t}, \quad t<\sigma_{k} .
$$

It is easy to see that $H_{1, k} \tilde{u} \in \mathscr{F}$ constitutes an $\mathscr{E}_{1}$-Cauchy sequence. Since we may take as $\tilde{u}$ a quasi-continuous version of $u$ in the restricted sense ([2]), we have $\lim _{t^{\prime} \uparrow t} \tilde{u}\left(X_{t^{\prime}}\right)=\tilde{u}\left(X_{t-}\right)$ for all $t \in[0, \infty), P_{x}$-a.s. for q.e. $x \in X$. Therefore the property (4.14) of the nest $\left\{F_{k}\right\}$ and the quasi-left continuity of the process $M$ imply

$$
\lim _{k \rightarrow \infty} H_{1, k} \tilde{u}(x)=E_{x}\left(e^{-\zeta} \tilde{u}\left(X_{\zeta}\right)\right)=0 \quad \text { q.e. } \quad x \in X,
$$

from which we conclude that $\lim _{k \rightarrow \infty} \mathscr{E}_{1}\left(H_{1, k} \tilde{u}, H_{1, k} \tilde{u}\right)=0$. We can now derive the desired equality (4.21) from (4.27) by letting $k$ tend to infinity and by using (4.14), Corollary to Theorem 2 and [4; Lemma 6].

The proof of the equivalence (i) $\Leftrightarrow$ (ii) has been completed. In order to prove the latter statement of Theorem 4, it suffices to show $A=0$ by assuming that

$$
\lim _{t \downarrow 0} \frac{1}{t} E_{v \cdot m}\left(A_{t}\right)=0 \quad \forall v \in \mathscr{F}_{k}, k=1,2, \cdots,
$$

for a CAF $A$ of bounded variation and for an increasing sequence $\left\{F_{k}\right\}$ of 
closed sets satisfying (4.14). Express $A$ as $A=A^{(1)}-A^{(2)}, A^{(i)} \in A_{c}^{+}, i=1$, 2, and consider $\nu^{(i)} \in S$ associated with $A^{(i)}, i=1,2$. Without loss of generality, we may suppose that $I_{F_{k}} \cdot \nu^{(i)} \in S_{0}, i=1,2, k=1,2, \cdots$. Then we have for each $k$

$$
\int_{F_{k}} \tilde{v}(x) \nu^{(1)}(d x)=\int_{F_{k}} \tilde{v}(x) \nu^{(2)}(d x)
$$

for any $v \in \mathscr{F}_{k}$ such that $v$ is a difference of 1-excessive functions. Hence in the same way as in the proof of the implication (i) $\Rightarrow$ (ii), we see that (4.30) holds for all $v \in \mathscr{F}_{k}$. Since any $f \in C_{0}(X)$ with $\operatorname{Supp}(f) \subset F_{k}$ can be approximated uniformly by $v \in \mathscr{F} \cap C_{0}(X)$ such that Supp [v] $\subset \operatorname{Supp}[f]$, we conclude from (4.30) that $I_{F_{k}} \cdot \nu^{(1)}=I_{F_{k}} \cdot \nu^{(2)}, k=1,2, \cdots$. We have $\nu^{(1)}=\nu^{(2)}$ because $\nu^{(i)}$ does not charges the exceptional set $X-\bigcup_{k=1}^{\infty} F_{k}$, $i=1,2$. Therefore $A=A^{(1)}-A^{(2)}=0$ completing the proof of Theorem 4 .

q.e.d.

COROLlaRY. The following conditions are equivalent each other for $u \in \mathscr{F}$ (resp. $u \in \mathscr{F}_{e}$ in transient case).

(i) $u$ is a difference of two 1-excessive functions in $\mathscr{F}$ (resp. 0-excessive functions in $\mathscr{F}_{e}$ ).

(ii) $u=U_{1}$ (resp. $u=U_{\nu}$ ) with a signed Radon measure $\nu$ of bounded 1order (resp. 0-order) energy integral.

(iii) $N^{[u]}$ is a CAF of bounded variation and the associated measure $\nu^{(1)}$ - $\nu^{(2)}$ in Theorem 4 has the additional property that $\lim _{k \rightarrow \infty}\left|\nu_{k}\right|$ is a positive Radon measure of bounded 1-order (resp. 0-order) energy integral.

In fact, relations (i) $\Leftrightarrow$ (ii) and (ii) $\Rightarrow$ (iii) are implied in [4; Lemma 4] and Lemma 2 respectively. (iii) $\Rightarrow$ (ii) is trivial.

Denote by $\mathscr{F}^{*}$ the dual space of the Dirichlet space $\mathscr{F}$ with metric $\mathscr{E}_{1}$. Each element $u \in \mathscr{F}$ defines a unique $T^{[u]} \in \mathscr{F} *$ by

$$
\mathscr{E}(u, v)=\left\langle T^{[u]}, v\right\rangle, \quad v \in \mathscr{F} .
$$

Sometimes it is convenient to express the relation (4.2) as

$$
\lim _{t \downarrow 0} \frac{1}{t} E_{v \cdot m}\left(A_{t}\right)=-\left\langle T^{[u]}, v\right\rangle, \quad \forall v \in \mathscr{F} .
$$

In transient case, the dual space $\mathscr{F}_{e}^{*}$ of the extended Dirichlet space $\left(\mathscr{F}_{e}, \mathscr{E}\right)$ plays a more specific role in characterizing the CAF's $N^{[u]}, u \in \mathscr{F}_{e}$ : 
THEOREM 5. Suppose the process $M$ is transient, then the family $\left\{N^{[u]}, u \in \mathscr{F}_{e}\right\}$ of CAF's stands in one-to-one correspondence with the dual space $\mathscr{F}_{e}^{*}$ of the extended Dirichlet space $\mathscr{F}_{e}$, the correspondence being characterized by the conditions that $A \in \mathcal{N}_{c}, \lim _{t ! 0} E_{x}\left(A_{t}\right)=0$ q.e. and

$$
\lim _{t \downarrow 0} \frac{1}{t} E_{v \cdot m}\left(A_{t}\right)=-\langle T, v\rangle, \quad v \in \mathscr{F}_{e} .
$$

Moreover $A$ is of bounded variation if and only if $T \in \mathscr{F}_{e}^{*}$ is expressible as

$$
T=\nu^{(1)}-\nu^{(2)}, \quad \nu^{(1)}, \nu^{(2)} \in S,
$$

in the sense of $(4.20)$.

In fact the relation (4.31) defines a one-to-one correspondence between $\mathscr{F}_{e}^{*}$ and $\mathscr{F}_{e}$ in this case. Hence Theorem 5 follows from Theorem 3 and Theorem 4.

\section{$\S 5$. Stochastic integrals}

We put

$$
\dot{A}_{c}=\left\{A-B: A, B \in A_{c}^{+}, E_{x}\left(A_{t}\right)<\infty, E_{x}\left(B_{t}\right)<\infty, \forall t>0 \text {, q.e. } x\right\} .
$$

For $M, L \in \mathscr{M}$, there exists a unique element $\langle M, L\rangle$ of $\dot{A}_{c}$ such that

$$
E_{x}\left(M_{t} L_{t}\right)=E_{x}\left(\langle M, L\rangle_{t}\right), \forall t>0 \text {, q.e. } \quad x \in X .
$$

This follows from the remark made in the paragraph after (1.5) and we may set $\langle M, L\rangle=\frac{1}{2}\{\langle M+L\rangle-\langle M\rangle-\langle L\rangle\}$.

Let us consider the family

$$
\mathscr{M}_{1}=\left\{M \in \mathscr{M}: \mu_{\langle M\rangle}(\in \boldsymbol{S}) \text { is a Radon measure }\right\} \text {. }
$$

Obviously $\mathscr{M}_{1}$ is a linear space containing our family $\mathscr{M}$. There exists for $M, L \in \mathscr{M}_{1}$ a unique signed Radon measure $\mu_{\langle M, L\rangle}$ such that

$$
\int_{X} f(x) \mu_{\langle M, L\rangle}(d x)=\lim _{t \backslash 0} \frac{1}{t} E_{m}\left(\int_{0}^{t} f\left(x_{s}\right) d\langle M, L\rangle_{s}\right), \quad \forall f \in C_{0}(X) .
$$

In fact, it suffices to put $\mu_{\langle M, L\rangle}=\frac{1}{2}\left\{\mu_{\langle M+L\rangle}-\mu_{\langle M\rangle}-\mu_{\langle L\rangle}\right\}$.

Lemma 3. If $M, L \in \mathscr{M}_{1}, f \in L^{2}\left(X ; \mu_{\langle M\rangle}\right)$ and $g \in L^{2}\left(X ; \mu_{\langle L\rangle}\right)$, then $f \cdot g$ is integrable with respect to the absolute variation $\left|\mu_{\langle M, L\rangle}\right|$ of $\mu_{\langle M, L\rangle}$ and 


$$
\left(\int_{X}|f \cdot g|\left|d \mu_{\langle M, L\rangle}\right|\right)^{2} \leqq \int_{X} f^{2} d \mu_{\langle M\rangle} \int_{X} g^{2} d \mu_{\langle L\rangle} .
$$

Proof. The proof is essentially the same as in Motoo-Watanabe [11, Lemma 10.1]. Making the expression $d \mu_{\langle M, L\rangle}=k_{1} d \nu, d \mu_{\langle M\rangle}=k_{2} d \nu$ and $d \mu_{\langle L\rangle}$ $=k_{3} d \nu$ with $\nu=\mu_{\langle M\rangle}+\mu_{\langle L\rangle}+\left|\mu_{\langle M, L\rangle}\right|$, we get from (5.2) and (5.4) $d \mu_{\langle a M+b L\rangle}=\left(a^{2} k_{2}\right.$ $\left.+2 a b k_{1}+b^{2} k_{3}\right) d \nu$ for any $a, b \in R$. Therefore the set $B_{0}=\bigcup_{a, b: \text { rational }}\{x \in X$; $\left.a^{2} k_{2}(x)+2 a b k_{1}(x)+b^{2} k_{3}(x)<0\right\}$ is $\nu$-negligible and we have for each $\alpha, \beta \in \boldsymbol{R}$ $\alpha^{2} f(x)^{2} k_{2}(x)+2 \alpha \beta\left|f(x) g(x) k_{1}(x)\right|+\beta^{2} g(x)^{2} k_{3}(x) \geqq 0 \quad$ for every $x \in X-B_{0}$. Integrating this with $\nu$,

$$
\alpha^{2} \int f^{2} d \mu_{\langle M\rangle}+2 \alpha \beta \int|f g|\left|d \mu_{\langle M, L\rangle}\right|+\beta^{2} \int g^{2} d \mu_{\langle L\rangle} \geqq 0,
$$

which proves (5.5).

q.e.d.

Theorem 6. Given $M \in \mathscr{M}_{1}$ and $f \in L^{2}\left(X ; \mu_{\langle M\rangle}\right)$, there exists a unique element $f \cdot M \in \mathscr{M}$ such that

$$
e(f \cdot M, L)=\frac{1}{2} \int_{X} f(x) \mu_{\langle M, L\rangle}(d x), \quad \forall L \in \mathscr{M} .
$$

The mapping $f \backsim f \cdot M$ is linear and continuous from $L^{2}\left(X ; \mu_{\langle M\rangle}\right)$ into the space $(\mathscr{M}, e)$.

Proof. By virtue of Lemma 3

$$
\left|\frac{1}{2} \int_{X} f d \mu_{\langle M, L\rangle}\right| \leqq \frac{1}{\sqrt{2}}\|f\|_{\left.L^{2}\left(\mu_{\langle M}\right\rangle\right)} \cdot \sqrt{e(L)}, \quad L \in \mathscr{M} .
$$

Hence Theorem 1 implies Theorem 6 together with the inequality

$$
\sqrt{e(f \cdot M)} \leqq \frac{1}{\sqrt{2}}\|f\|_{L^{2}(\mu\langle\langle M\rangle} .
$$

$f \cdot M \in \mathscr{M}$ constructed in Theorem 6 is called the stochastic integral. This terminology is legitimate in view of the following theorem.

TheOREM 7. Let $M, f$ and $f \cdot M$ be as in Theorem 6 . Then

$$
d \mu_{\langle f \cdot M, L\rangle}=f \cdot d \mu_{\langle M, L\rangle}, \quad \forall L \in \mathscr{M} .
$$

Moreover the following approximation holds for $f \in C_{0}(X)$ :

$$
\lim _{|\Delta| \rightarrow 0} E_{x}\left(\left\{(f \cdot M)_{t}^{(\Delta)}-(f \cdot M)_{t}\right\}^{2}\right)=0, \quad t>0, \text { q.e. } x
$$


where

$$
(f \cdot M)_{t}^{\langle\Delta)}=\sum_{i=1}^{n} f\left(X_{t_{i-1}}\right)\left(M_{t_{i}}-M_{t_{i-1}}\right),
$$

$\Delta$ denotes the partition $0=t_{0}<t_{1}<\cdots<t_{n}=t$ and $|\Delta|=\max _{1 \leqq i \leqq n}\left(t_{i}-t_{i-1}\right)$.

Proof. Let $B$ be a common proper exceptional set for $M$ and $\langle M\rangle$ such that the relation (1.6) holds for every $x \in X-B$. Applying the same argument as in P. A. Meyer [10; III, Théorème 4] to the Hunt process $\left.\boldsymbol{M}\right|_{X-B}$ and its AF's $M$ and $\langle M\rangle$ in the ordinary sense, we can see for $f \in C_{0}(X)$ that there exists a unique martingale $\mathrm{AF} \tilde{M}$ of $\boldsymbol{M}_{X-B}$ in the ordinary sense such that

$$
\lim _{|\Delta| \rightarrow 0} E_{x}\left(\left\{(f \cdot M)_{t}^{(\Delta)}-\tilde{M}_{t}\right\}^{2}\right)=0, \quad x \in X-B .
$$

Furthermore

$$
E_{x}\left(\tilde{M}_{t}^{2}\right)=E_{x}\left(\int_{0}^{t} f\left(X_{s}\right)^{2} d\langle M\rangle_{s}\right), \quad x \in X-B
$$

It follows from this that $e(\tilde{M})=\lim _{t \downarrow 0} \frac{1}{2 t} E_{m}\left(\tilde{M}_{t}^{2}\right)=\frac{1}{2} \int_{X} f^{2} d \mu_{\langle M\rangle}<\infty$ and consequently $\tilde{M} \in \mathscr{M}$.

On the other hand (5.2) and (5.12) imply

$$
E_{x}\left(\langle\tilde{M}, L\rangle_{t}\right)=E_{x}\left(\int_{0}^{t} f\left(X_{s}\right) d\langle M, L\rangle_{t}\right)
$$

for any $L \in \mathscr{M}$ and q.e. $x \in X$. When $L \in \mathscr{M}, \mu_{\langle\tilde{M}, L\rangle}$ is a bounded signed measure and hence $\lim _{t ! 0} \frac{1}{2 t} E_{h \cdot m}\left(\langle\tilde{M}, L\rangle_{t}\right)=\int_{X} h(x) \mu_{\langle\tilde{M}, L\rangle}(d x)$ for any bounded $\gamma$-excessive function $h$ in view of the relation (0.3). Therefore we have from (5.14)

$$
\int_{X} h(x) \mu_{\langle\tilde{M}, L\rangle}(d x)=\int_{X} h(x) f(x) \mu_{\langle M, L\rangle}(d x), \quad L \in \mathscr{M} .
$$

By setting $h=\alpha R_{\alpha} \tilde{h}, \tilde{h} \in C_{0}(X)$, and letting $\alpha$ tend to infinity, we conclude that (5.15) holds for any $h \in C_{0}(X)$. Therefore $d \mu_{\langle\tilde{M}, L\rangle}=f \cdot d \mu_{\langle M, L\rangle}, \forall L \in \mathscr{M}$. In particular $e(\tilde{M}, L)=\frac{1}{2} \int_{X} f d_{\mu_{\langle M, L\rangle}}$ which means $\tilde{M}=f \cdot M$.

We have proved Theorem 7 when $f \in C_{0}(X)$. But on account of inequalities (5.7) and (5.8), the relation (5.15) for $\tilde{M}=f \cdot M$ readily extends 
to $f \in L^{2}\left(X ; \mu_{\langle M\rangle}\right)$. The proof of Theorem 7 is completed.

q.e.d.

Corollary (i) For $M \in \mathscr{M}_{1}, f \in L^{2}\left(X ; \mu_{\langle M\rangle}\right)$ and $g \in L^{2}\left(X ; f^{2} \mu_{\langle M\rangle}\right)$,

$$
g \cdot(f \cdot M)=g f \cdot M .
$$

(ii) For $M, L \in \mathscr{M}_{1}, f \in L^{2}\left(X ; \mu_{\langle M\rangle}\right)$ and $g \in L^{2}\left(X ; \mu_{\langle L\rangle}\right)$,

$$
e(f \cdot M, g \cdot L)=\frac{1}{2} \int_{X} f(x) g(x) \mu_{\langle M, L\rangle}(d x) .
$$

Our stochastic integral $f \cdot M$ can be reduced to the stochastic integral due to Motoo-Watanabe [11] in the following way. Suppose that $M \in \mathscr{M}$ and $f \in L^{2}\left(X ; \mu_{\langle M\rangle}\right)$. Then

$$
E_{x}\left(\int_{0}^{t} f\left(X_{s}\right)^{2} d\langle M\rangle_{s}\right)<\infty \text { q.e. } \quad x \in X .
$$

In fact we have by Lemma 1

$$
E_{\nu}\left(\int_{0}^{t} f\left(X_{s}\right)^{2} d\langle M\rangle_{s}\right) \leqq(1+t)\left\|U_{1} \nu\right\|_{\infty} \cdot \int_{X} f(x)^{2} \mu_{\langle M\rangle}(d x)<\infty \quad \text { for any } \nu \in S_{00} \text {. }
$$

Let $B$ be a common proper exceptional set for $M$ and $\langle M\rangle$ such that (1.6) and (5.18) hold for every $x \in X-B$. According to a general theorem [10; III, Théorème 4] applied to the Hunt process $\left.\boldsymbol{M}\right|_{X-B}$, the stochastic integral $\widetilde{f \cdot M}$ in the sense of Motoo-Watanabe is well defined. We claim for $M \in \mathscr{M}_{1}$

$$
\widetilde{f \cdot M}=f \cdot M
$$

the equality being understood in the present sense of the equivalence of AF's.

Indeed (5.19) for $f \in C_{0}(X)$ has been shown in the proof of the preceding theorem. It is then easy to extend the relation (5.19) to general $f$ as above.

We exhibit in the next section examples of $f \in L^{2}\left(X ; \mu_{\langle M\rangle}\right)$ for which $E_{x}\left(\int_{0}^{t} f\left(X_{s}\right)^{2} d\langle M\rangle_{s}\right)=\infty$ for some $x$. For such $f$, the stochastic integral $f \cdot M$ in our sense is well defined, while the stochastic integral $\widetilde{f} \cdot M$ in Motoo-Watanabe sense can not be defined unless we admit an exceptional set $B$ as above.

In the remainder of this section, we study some properties of the 
stochastic integral $f \cdot M^{[u]}$ relevant to the martingale $\mathrm{AF} M^{[u]} \in \mathscr{\mathscr { M }}$ constructed in $\S 3$ for $u \in \mathscr{F}$.

THEOREM 8. The family $\left\{f \cdot M^{[v]}\right\}$ of stochastic integrals is dense in the space $(\mathscr{M}, e)$, provided that $f$ (resp. u) runs over a uniformly dense subfamily of $C_{0}(X)$ (resp. an $\mathscr{E}_{1}$-dense subfamily of $\mathscr{F}$ ).

Proof. Suppose that $M \in \mathscr{M}$ is orthogonal to the family $\left\{f \cdot M^{[u]}\right\}$ in the statement of Theorem 8: $e\left(M, f \cdot M^{[u]}\right)=0$. Then by (5.6) $\int f(x) \mu_{\langle M, M[u]\rangle}(d x)$ $=0$, which in turn extends to all $u \in \mathscr{F}$ by virtue of (3.2) and (5.7). Therefore $\mu_{\langle M, M[u]\rangle}=0$ and hence $\left\langle M, M^{[u]}\right\rangle=0$ for any $u \in \mathscr{F}$.

By setting $u=R_{\alpha} g, g \in C_{0}(X)$, we can conclude $M=0$ on account of Motoo-Watanabe [11; Theorem 12.2].

q.e.d.

We now express the energy of the stochastic integral in terms of the Dirichlet form.

Lemma 4. For $u \in \mathscr{F}_{b}$ and a quasi-continuous function $f \in \mathscr{F}_{b}$,

$$
\int_{X} f(x) \mu_{\langle M[u]\rangle}(d x)=2 \mathscr{E}^{\mathscr{r} \mathrm{res}}(u \cdot f, u)-\mathscr{E}^{\mathrm{res}}\left(u^{2}, f\right) .
$$

Proof. By (0.3) and (1.6),

$$
\int_{X} f(x) \mu_{\langle M[u]\rangle}(d x)=\lim _{t \downarrow 0} \frac{1}{t} E_{f \cdot m}\left(\left(M_{t}^{[u]}\right)^{2}\right)
$$

when $f$ is a bounded $\gamma$-excessive function in $\mathscr{F}$. Since $M_{t}^{[u]}=\tilde{u}\left(X_{t}\right)$ $-\tilde{u}\left(X_{0}\right)-N_{t}^{[u]}$ and $E_{f \cdot m}\left(\left(N_{t}^{[u]}\right)^{2}\right) \leqq\|f\|_{\infty} E_{m}\left(\left(N_{t}^{[u]}\right)^{2}\right)$, we can see that the right hand side of (5.21) equals $\lim _{t \downarrow 0} \frac{1}{t} E_{f \cdot m}\left(\left(u\left(X_{t}\right)-u\left(X_{0}\right)\right)^{2}\right)=\lim _{t \downarrow 0}\left\{\frac{2}{t}(u f\right.$, $\left.\left.u-p_{t} u\right)-\frac{1}{t}\left(f-p_{t} f, u^{2}\right)-\frac{1}{t}\left(u^{2} f, 1-p_{t} 1\right)\right\}=2 \mathscr{E}(u f, u)-\mathscr{E}\left(f, u^{2}\right)-\int_{X} u^{2} f d k$, which can be rewritten as (5.20).

(5.20) is now valid if $f$ is replaced by $\alpha R_{\alpha} f, f \in \mathscr{F}_{b}$. After letting $\alpha$ tend to infinity, we get (5.20) for general $f \in \mathscr{F}_{b}$. q.e.d.

Corollary 1. For any $u, v \in \mathscr{F}_{c}$ and a quasi-continuous function $f$ $\in \mathscr{F}_{b}$

$$
\int_{X} f \mu_{\langle M[u], M[v]\rangle}(d x)=\mathscr{E}^{\mathrm{res}}(u f, v)+\mathscr{E}^{\mathrm{res}}(v f, u)-\mathscr{E}^{\mathrm{res}}(u v, f) .
$$


By making use of the relation (5.17), we further have

Corollary 2. For $u_{1}, u_{2} \in \mathscr{F}_{b}$ and quasi-continuous functions $f_{1}, f_{2} \in \mathscr{F}_{b}$, the stochastic integrals $f_{1} \cdot M^{\left[u_{1}\right]}, f_{2} \cdot M^{\left[u_{2}\right]} \in \mathscr{M}$ are well defined and

$$
2 e\left(f_{1} \cdot M^{\left[u_{1}\right]}, f_{2} \cdot M^{\left[u_{2}\right]}\right)=\mathscr{E}^{\mathscr{E r e s}}\left(u_{1} f_{1} f_{2}, u_{2}\right)+\mathscr{E}^{\text {res }}\left(u_{2} f_{1} f_{2}, u_{1}\right)-\mathscr{E}^{\text {res }}\left(u_{1} u_{2}, f_{1} f_{2}\right)
$$

M. L. Silverstein [12; Theorem 11.3] first singled out the measure $\mu_{\langle M[u]\rangle}$ for $u \in \mathscr{F}_{b}$ through the formula (5.20). For example, if $\mathscr{E}$ is a Dirichlet form on $L^{2}\left(R^{n}\right)$ given by

$$
\mathscr{E}(u, v)=\sum_{i, j=1}^{n} \int_{R^{n}} \frac{\partial u}{\partial x_{i}} \frac{\partial v}{\partial x_{j}} \nu_{i j}(d x), \quad u, v \in C_{0}^{\infty}\left(\boldsymbol{R}^{n}\right),
$$

then it holds that $d_{\mu_{M\langle[n], M[v]\rangle}}=2 \sum_{i, j=1}^{n} \frac{\partial u}{\partial x_{i}} \frac{\partial v}{\partial x_{j}} \nu_{i j}, u, v \in C_{0}^{\infty}\left(\boldsymbol{R}^{n}\right)$.

Using the formula (5.20), Yves Lejan [8] then derived the following transformation rule of the energy measure: in case that the process $M$ is continuous

$$
d \mu_{\langle M[\Phi(u)], M[v]\rangle}=\sum_{i=1}^{n} \Phi_{x_{i}}(\boldsymbol{u}) d \mu_{\left\langle M\left[u_{i}\right], M[v]\right\rangle}, \quad v \in \mathscr{F}_{b},
$$

where $\boldsymbol{u}=\left(u_{1}, u_{2}, \cdots, u_{n}\right)$ is a collection of quasi-continuous functions in $\mathscr{F}_{b}, \Phi$ is $C^{1}$-function on $\boldsymbol{R}^{n}$ vanishing at the origin and possessing bounded derivatives. An integration of $f \in C_{0}(X)$ by means of the both hand sides of (5.22) leads us to

$$
e\left(M^{[\Phi(u)]}, f \cdot M^{[v]}\right)=\sum_{i=1}^{n} e\left(\Phi_{x_{i}}(\boldsymbol{u}) \cdot M^{\left[u_{i}\right]}, f \cdot M^{[v]}\right), \quad v \in \mathscr{F}_{b},
$$

with the aid of (5.17). Hence, by Theorem 8, we arrive at the following version of Ito's formula.

Theorem 9. Suppose that the process $\boldsymbol{M}$ is continuous. Let $\boldsymbol{u}=\left(u_{1}\right.$, $u_{2}, \cdots, u_{n}$ ) and $\Phi$ be as above. Then the composite function $\Phi(\boldsymbol{u})$ belongs to $\mathscr{F}_{b}$ again and

$$
M^{[\Phi(\boldsymbol{u})]}=\sum_{i=1}^{n} \Phi_{x_{i}}(\boldsymbol{u}) \cdot M^{\left[u_{i}\right]}
$$

\section{§6. Case of the Brownian motion}

Applying the general theorems obtained so far, we examine in this section the case that $\boldsymbol{M}=\left(X_{t}, P_{x}\right)$ is the Brownian motion on $\boldsymbol{R}^{n}$. In 
particular we represent the space $\mathscr{\mathscr { M }}$ by means of the stochastic integrals and thereby generalize Ito's formula.

The associated Dirichlet space on $L^{2}\left(\boldsymbol{R}^{n}\right)$ is the Sobolev space

$$
H^{1}\left(\boldsymbol{R}^{n}\right)=\left\{u \in L^{2}\left(\boldsymbol{R}^{n}\right): \frac{\partial u}{\partial x_{i}} \in L^{2}\left(\boldsymbol{R}^{n}\right), 1 \leqq i \leqq n\right\}
$$

with

$$
\mathscr{E}(u, v)=\frac{1}{2} \sum_{i=1}^{n} \int_{R_{n}} \frac{\partial u}{\partial x_{i}} \frac{\partial v}{\partial x_{i}} d x
$$

the derivatives being taken in the sense of Schwartz distribution. When $n \geqq 3, M$ is transient and the associated extended Dirichlet space $\left(\mathscr{F}_{e}, \mathscr{E}\right)$ is the completion of $C_{0}^{\infty}\left(R^{n}\right)$ by means of the Dirichlet integral. The quasi-continuous function in $\mathscr{F}_{e}$ is called the BLD function of potential type. In view of the Sobolev inequality $\|u\|_{p_{0}} \leqq C \sqrt{\mathscr{E}(u, u)}, u \in C_{0}^{\infty}\left(\boldsymbol{R}^{n}\right)$, $\frac{1}{p_{0}}=\frac{1}{2}-\frac{1}{n}$, we can regard the space $\mathscr{F}_{e}$ as a closed subspace of

$$
\mathscr{G}=\left\{u \in L_{1 \mathrm{loc}}^{1}\left(\boldsymbol{R}^{n}\right): \frac{\partial u}{\partial x_{i}} \in L^{2}\left(\boldsymbol{R}^{n}\right), 1 \leqq i \leqq n\right\}
$$

with inner product (6.2).

Let $X_{t}^{(i)}$ be the $i$-th coordinate of $X_{t}$ and put $B_{t}^{(i)}=X_{t}^{(i)}-X_{0}^{(i)}, t \geqq 0$, $1 \leqq i \leqq n$. Then $B^{(i)} \in \mathscr{M}_{1}$ with

$$
\left\langle B^{(i)}, B^{(j)}\right\rangle_{t}=\delta_{i j} \cdot t, \quad \mu_{\langle B(i)\rangle}(d x)=d x .
$$

Therefore the stochastic integral $f \cdot B^{(i)} \in \mathscr{M}$ in the sense of $\S 5$ is well defined for any $f \in L^{2}\left(\boldsymbol{R}^{n}\right)$. According to a remark made in $\S 5$, any Borel function $f \in L^{2}\left(\boldsymbol{R}^{n}\right)$ satisfies

$$
E_{x}\left(\int_{0}^{t} f\left(X_{s}\right)^{2} d s\right)<\infty
$$

for q.e. $x \in \boldsymbol{R}^{n}$ and the stochastic integral $f \cdot B^{(i)}$ coincides with the ordinary one of Motoo-Watanabe with respect to the Brownian motion $\boldsymbol{M}$ retsricted to $\boldsymbol{R}^{n}-B, B$ being some Borel polar set.

For instance the function in $L^{2}\left(\boldsymbol{R}^{n}\right)$

$$
f(x)=f_{0}(x) /|x|^{\alpha}, \quad \alpha<\frac{n}{2}, \quad f_{0} \in C_{0}\left(R^{n}\right)
$$


satisfies (6.5) for all $x \in R^{n}$ if $\alpha<1$. In this case the AF $f \cdot B^{(i)}$ in the sense of Motoo-Watanabe can be well defined for every starting point $x \in R^{n}$. When $\alpha \geqq 1$ however, (6.5) is violated for $x=0$ and the origin 0 must be excluded as the exceptional set for the AF $f \cdot B^{(i)}$.

TheOREm 10. (i) The space $\mathscr{M}$ of $M A F$ 's of finite energy can be expressed as

$$
\mathscr{M}=\left\{M=\sum_{i=1}^{n} f_{i} \cdot B^{(i)}: f \in L^{2}\left(\boldsymbol{R}^{n}\right), 1 \leqq i \leqq n\right\}
$$

and the following isometry between the space $\mathscr{M}$ and vector fields $\left(f_{1}, f_{2}\right.$, $\left.\cdots, f_{n}\right)$ holds:

$$
e(M)=\frac{1}{2} \sum_{i=1}^{n}\left\|f_{i}\right\|_{L^{2}\left(R^{n}\right)}^{2}
$$

(ii) $M \in \mathscr{M}$ equals $M^{[u]}$ for some $u \in H^{1}\left(\boldsymbol{R}^{n}\right)\left(u \in \mathscr{F}_{e}\right.$ in case $\left.n \geqq 3\right)$ if and only if the vector field corresponding to $M$ is $\operatorname{grad} u$ :

$$
M^{[u]}=\operatorname{grad} u \cdot B\left(=\sum_{i=1}^{n} \frac{\partial u}{\partial x_{i}} \cdot B^{(i)}\right) .
$$

(iii) When $n \geqq 3$, the extended Dirichlet space $\left(\mathscr{F}_{e}, \mathscr{E}\right)$ is identified by the mapping $u \backsim \operatorname{grad} u \cdot B$ with a closed subspace of $(\mathscr{M}, e)$.

Proof. We first prove the relation (6.8) for $u \in C_{0}^{2}\left(\boldsymbol{R}^{n}\right)$. In this case, Ito formula gives

$$
u\left(X_{t}\right)-u\left(X_{0}\right)=\sum_{i=1} \int_{0}^{t} \frac{\partial u}{\partial x_{i}}\left(X_{s}\right) d B_{s}^{(i)}+\frac{1}{2} \int_{0}^{t} \Delta u\left(X_{s}\right) d s
$$

which holds $P_{x}$-a.e. for every $x \in R^{n}$. Since the first term on the right hand side is a version of the stochastic integral (grad $u \cdot B)_{t}$ in our sense and the second term belongs to $\mathscr{N}_{c}$, we are led to (6.8) with the help of the uniqueness of the decomposition (3.1).

We know from Theorem 8 that the family $\left\{f \cdot M^{[u]}: f \in C_{0}\left(\boldsymbol{R}^{n}\right), u \in C_{0}^{2}\left(\boldsymbol{R}^{n}\right)\right\}$ spans the space $(\mathscr{M}, e)$. By the above observation and (5.17), each member $f \cdot M^{[u]}$ of the family can be expressed as the stochastic integral $\sum_{i=1}^{n}\left(f \cdot \frac{\partial u}{\partial x_{i}}\right) \cdot B^{(i)}$ based on $B^{(i)}$ 's. Hence any $M \in \mathscr{M}$ can be approximated by a sequence $\left\{M^{(k)}=\sum_{i=1}^{n} f_{i}^{(k)} \cdot B^{(i)}: f_{i}^{(k)} \in L^{2}\left(R^{n}\right), k=1,2, \cdots\right\}$ with $e$-metric. 
On the other hand (5.18) and (6.4) imply

$$
e\left(M^{(k)}-M^{(\ell)}\right)=\frac{1}{2} \sum_{i=1}^{n}\left\|f_{i}^{(k)}-f_{i}^{(\ell)}\right\|_{L^{2}\left(\boldsymbol{R}^{n}\right)}^{2} .
$$

Denote by $f_{i}$ the $L^{2}$-limit of $f_{i}^{(k)}, 1 \leqq i \leqq n$. Then we have $M=\sum_{i=1}^{n} f_{i} \cdot B^{(i)}$ together with the equality (6.7).

If $u \in H^{1}\left(R^{n}\right)\left(u \in \mathscr{F}_{e}\right.$ when $\left.n \geqq 3\right)$, we can select $u^{(k)} \in C_{0}^{2}\left(\boldsymbol{R}^{n}\right)$ which is $\mathscr{E}$-convergent to $u$. By the continuity embodied in (3.2), $M^{[u(k)]}$ is then $e$-convergent to $M^{[u]}$. Since derivatives $\frac{\partial u^{(k)}}{\partial x_{i}}$ are $L^{2}$-convergent to $\frac{\partial u}{\partial x_{i}}$, we see as above that $\operatorname{grad} u^{(k)} \cdot B$ is $e$-convergent to $\operatorname{grad} u \cdot B$. We thus extend the identity (6.8) for the present $u$.

(iii) follows from Corollary 2 to Theorem 2. q.e.d.

Let $\rho_{\varepsilon}$ be a mollifier: $\rho_{\varepsilon}$ is a non-negative $C^{\infty}$-function vanishing when $|x| \geqq \varepsilon$ and $\int_{R^{n}} \rho_{\varepsilon}(x) d x=1$. The additive functional

$$
\boldsymbol{t}_{\mathrm{s}}(t, y)=\frac{1}{2} \int_{0}^{t} \rho_{\varepsilon}\left(X_{s}-y\right) d s
$$

expresses a normalized sojourn time of the Brownian path near the point $y$. We then have for $u \in L_{\mathrm{loc}}^{1}\left(\boldsymbol{R}^{n}\right)$ and $u_{\mathrm{s}}(x)=\rho_{\varepsilon}^{*} u(x), x \in \boldsymbol{R}^{n}$,

$$
\frac{1}{2} \int_{0}^{t} \Delta u_{\varepsilon}\left(X_{s}\right) d s=\left\langle\boldsymbol{t}_{\mathrm{s}}(t, \cdot), \Delta u\right\rangle
$$

$\Delta$ on the right hand side being taken in the sense of Schwartz distribution.

We now state an extension of Ito's formula in our direction.

Theorem 11. Let $u \in H^{1}\left(\boldsymbol{R}^{n}\right)\left(u \in \mathscr{F}_{e}\right.$ when $\left.n \geqq 3\right)$.

(i) Let $\tilde{u}$ be a quasi-continuous version of $u$. Then the identity

$$
\tilde{u}\left(X_{t}\right)-\tilde{u}\left(X_{0}\right)=(\operatorname{grad} u \cdot B)_{t}+N_{t}^{[u]}
$$

holds with

$$
N_{t}^{[u]}=\lim _{\varepsilon_{n} \downarrow 0} \frac{1}{2} \int_{0}^{t} \Delta u_{\varepsilon_{n}}\left(X_{s}\right) d s .
$$

Here the convergence is uniform in $t$ on each finite interval $P_{x}$-a.s. for q.e. $x \in \boldsymbol{R}^{n} . \quad\left\{\varepsilon_{n}\right\}$ is some sequence decreasing to 0 and depending only on the function $u$. 
(ii) $N^{[u]}$ is characterized as a unique element of $\mathscr{N}_{c}$ such that $\lim _{t \downarrow 0} E_{x}\left(N_{t}^{[u]}\right)$ $=0$ q.e. and

$$
\lim _{t \downarrow 0} \frac{1}{t} \int_{\boldsymbol{R}^{n}} E_{x}\left(N_{t}^{[u]}\right) \phi(x) d x=\left\langle\frac{1}{2} \Delta u, \phi\right\rangle, \quad \forall \phi \in C_{0}^{\infty}\left(\boldsymbol{R}^{n}\right) .
$$

(iii) When $n \geqq 3$, the relation (ii) determines a one-to-one correspondence between the family $\left\{N^{[u]}, u \in \mathscr{F}_{e}\right\}$ of $C A F$ of zero energy and the family $\left\{\frac{1}{2} \Delta u, u \in \mathscr{F}_{e}\right\}$ of distributions. The latter family exhausts the dual space $\mathscr{F}_{e}^{*}$ of $\mathscr{F}_{e}$ described explicitly as follows:

$$
\mathscr{F}_{e}^{*}=\left\{T: \text { tempered distribution, } \hat{T} \in L^{2}\left(|\xi|^{-2} d \xi\right)\right\}
$$

where $\hat{T}$ denotes the Fourier transform.

(iv) $N^{[u]}$ is of bounded variation if and only if the distribution $-\frac{1}{2} \Lambda u$ is represented by a difference $\nu^{(1)}-\nu^{(2)}$ of some smooth measures $\nu^{(1)}$ and $\nu^{(2)}$ in the manner of (4.19). In particular if $\nu^{(1)}$ and $\nu^{(2)}$ are positive Radon measures or more generally if a common nest $\left\{F_{k}\right\}$ associated with $\nu^{(1)}$ and $\nu^{(2)}$ can be chosen in such a way that each $F_{k}$ is compact and contained in the interior ${\stackrel{\circ}{F_{k+1}}}_{i}$ of $F_{k+1}$, then

$$
N_{t}^{[u]}=\lim _{\varepsilon_{n} \downarrow 0} \int_{R^{n}} \boldsymbol{t}_{\varepsilon_{n}}(t, y) \nu(d y), \quad \nu=\nu^{(2)}-\nu^{(1)},
$$

the convergence being in the same sense as in (6.14).

Proof. (i) is a consequence of Theorem 2, its Corollary and Theorem 9. Since $u_{\varepsilon}$ converges as $\varepsilon \downarrow 0$ to $u$ with respect to the Dirichlet integral, Corollary 1 (ii) to Theorem 2 implies that $N_{t}^{\left[u_{\varepsilon}\right]}=\frac{1}{2} \int_{0}^{t} \Lambda u_{\varepsilon}\left(X_{s}\right) d s$ is convergent to $N_{t}^{[u]}$ in the sense of the statement of the theorem.

(ii), (iii) and (iv) are applications to the Brownian motion of Theorem 3, Theorem 4 and Theorem 5 respectively. Suppose that a nest $\left\{F_{k}\right\}$ associated with $\nu=\nu^{(2)}-\nu^{(1)}$ satisfies the property stated in (iv). For q.e. starting point $x$ and $P_{x}$-a.s., $\omega \in \Omega$, the set $\left\{X_{s}(\omega), 0 \leqq s \leqq t\right\}$ is then contained in some $F_{k}$. Consequently $\operatorname{Supp}\left[\boldsymbol{t}_{\mathrm{s}}(t, \cdot)\right] \subset F_{k+1}$ for sufficiently small $\varepsilon>0$ and hence the relation (4.19) yields

$$
\left\langle\boldsymbol{t}_{\varepsilon}(t, \cdot), \Delta u\right\rangle=\int_{\boldsymbol{R}^{n}} \boldsymbol{t}_{\varepsilon}(t, x) \nu(d x) .
$$

Combining (6.14) with (6.12), we arrive at (6.15).

q.e.d. 
In general we can not reduce the difference of smooth measures in the statement (iv) of the above theorem to a difference of positive measures of finite energy integrals. For instance consider a signed measure $f(x) d x$ on $R^{3}$ with

$$
f(x)= \begin{cases}|x|^{\alpha} \sin \frac{1}{|x|}, & |x|<1 \\ 0 \quad, & |x| \geqq 1 .\end{cases}
$$

$f(x) d x$ can always be expressed as a difference $f^{+}(x) d x-f(x) d x$ of smooth measures and

$$
A_{t}=\int_{0}^{t} f\left(X_{t}\right) d s
$$

defines a CAF of bounded variation of the 3-dimensional Brownian motion with a possible exceptional set being the origin 0 (see [4]). The 0-order resolvent is now given by the Newtonian kernel $g(x, y)=\frac{1}{4 \pi} \frac{1}{|x-y|}$ and the limit

$$
u(x)=\lim _{\varepsilon \nmid 0} \int_{|y|>\varepsilon} g(x, y) f(y) d y
$$

converges for every $x \in \boldsymbol{R}^{3}$ whenever $\alpha>-3$. The Dirichlet integral of $u$ equals

$$
\lim _{\varepsilon \backslash 0} \int_{|x|>\varepsilon,|y|>\varepsilon} g(x, y) f(x) f(y) d x d y .
$$

When $-3<\alpha,(6.19)$ is convergent and consequently, $u$ of (6.18) belongs to $\mathscr{F}_{e}$. Moreover $-N^{[u]}$ is given by (6.17) on account of Corollary to Theorem 2. When $-3<\alpha \leqq-\frac{5}{2}$ however, (6.19) is not absolutely convergent, which means that $-\frac{1}{2} \Delta u=f d x$ can not be expressed as a difference of positive Radon measures of finite energy integrals. In the last case, $u$ can neither be expressed as a difference of excessive functions belonging to $\mathscr{F}_{e}$ (Corollary to Theorem 4).

In the case of the one-dimensional Brownian motion, non-empty exceptional set is absent. This considerably simplify the situation. The quasi-continuity and the smooth measure reduce to the ordinary continuity and the positive Radon measure respectively. In particular Theorem 11 now reads as follows: 
TheORem 12. Let $n=1$ and $u \in H^{1}\left(\boldsymbol{R}^{1}\right)$.

(i ) $u\left(X_{t}\right)-u\left(X_{0}\right)=\int_{0}^{t} u^{\prime}\left(X_{s}\right) d B_{s}+N_{t}^{[u]} P_{x}$-a.s. $\forall x \in R^{1}$.

Here the first term on the right hand side is the stochastic integral in the ordinary sense of Motoo-Watanabe and $N_{t}^{[u]}$ is given by (6.14), the convergence being uniform in $t$ on each finite interval $P_{x}$-a.s. $\forall x \in \boldsymbol{R}^{n}$.

(ii) $N^{[u]}$ is characterized as a unique element of $\mathscr{N}_{c}$ such that $\lim _{t \downarrow 0} E_{x}\left(N_{t}^{[u]}\right)$ $=0, x \in R^{1}$, and

$$
\lim _{t \downarrow 0} \frac{1}{t} \int_{-\infty}^{\infty} E_{x}\left(N_{t}^{[u]}\right) \phi(x) d x=\left\langle\frac{1}{2} u^{\prime \prime}, \phi\right\rangle, \quad \forall \phi \in C_{0}\left(\boldsymbol{R}^{1}\right) .
$$

(iii) $N^{[u]}$ is of bounded variation if and only if the distribution $\frac{1}{2} u^{\prime \prime}$ is a (signed) Radon measure or equivalently $u^{\prime}$ is of bounded variation on each finite interval. When this is the case, $N_{t}^{[u]}$ is expressed by the measure $\nu=\frac{1}{2} u^{\prime \prime}$ as the local time integral:

$$
N_{t}^{[u]}=\int_{R^{1}} t(t, y) \nu(d y), \quad P_{x^{-} \text {-a.s }}
$$

where $t(t, y)$ is the local time of the Brownian path.

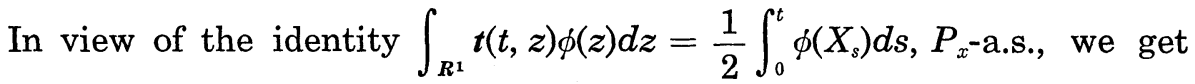
$\boldsymbol{t}_{\varepsilon}(t, y)=\int_{R^{1}} t(t, z) \rho_{\varepsilon}(z-y) d z$ which converges as $\varepsilon \downarrow 0$ to $t(t, y)$. Hence (6.20) follows from the expression (6.15).

As an example of Theorem 12 (iii), consider a function $g \in C_{0}\left(R^{1}\right)$ satisfying $\int_{R^{1}} g(x) d x=0$ and set $u(x)=\int_{-\infty}^{x} g(x) d x$. Then $N^{[u]}$ is of bounded variation if and only if the function $g$ is of bounded variation.

\section{Appendices}

\section{(I) Killing measure and resurrected Dirichlet form}

Proposition 7.1. There exists a unique positive Radon measure $k$ charging no exceptional set such that

$$
\lim _{t \downarrow 0} \frac{1}{t} \int_{\tilde{K}} u(x)^{2}\left(1-p_{t} 1(x)\right) m(d x)=\int_{X} \tilde{u}(x)^{2} k(d x)
$$

for any $u \in \mathscr{F}$. It holds that 


$$
E_{h \cdot m}\left(f\left(X_{\zeta-}\right) ; \zeta \leqq t\right)=\int_{0}^{t}\left\langle f k, p_{s} h\right\rangle d s
$$

for any $f, h \in \mathscr{B}^{+}$and $t>0$. In particular $E_{x}\left(e^{-\alpha \zeta} f\left(X_{\zeta_{-}}\right)\right)$is a quasi-continuous version of the potential $U_{\alpha}(f \cdot k)$ when $\alpha>0$ and $f$ is of compact support.

We call the measure $k$ the killing measure of the process $\boldsymbol{M}$.

Proof. In view of the formula

$$
\begin{gathered}
\frac{1}{2 t} \int_{X \times X}(u(x)-u(y))^{2} p_{t}(x, d y) m(d x)+\frac{1}{t} \int_{X} u(x)^{2}\left(1-p_{t} 1(x)\right) m(d x) \\
=\frac{1}{t}\left(u, u-p_{t} u\right) \uparrow \mathscr{E}(u, u), \quad t \downarrow 0,
\end{gathered}
$$

and the regularity of the Dirichlet space, we can subtract a sequence $t_{n} \downarrow 0$ such that the measure $\frac{1}{t_{n}}\left(1-p_{t_{n}} 1(x)\right) m(d x)$ converges vaguely to a positive Radon measure $k$ on $X$ and

$$
\int_{X} u(x)^{2} k(d x) \leqq \mathscr{E}(u, u), \quad u \in \mathscr{F} \cap C_{0}(X)
$$

In particular $I_{K} \cdot k$ is of finite energy integral for any compact set $K$ and hence $k$ is smooth.

By making use of (7.3) and (7.4) again, we can easily see that the equality (7.1) holds provided that we take the limit of the left hand side only along the sequence $\left\{t_{n}\right\}$. Therefore it suffices to show (7.2) because the measure $k$ is then independent of the choice of $\left\{t_{n}\right\}$.

Take non-negative $f, g \in C_{0}(X)$ and we first show (7.2) for $f$ and $h=\alpha R_{\alpha} g$. Notice that $h \in \mathscr{D}(A) \cap L^{1}(X ; m), A$ being the infinitesimal generator of the $L^{2}$-semigroup determined by $p_{t}$. We have then

$$
\begin{aligned}
E_{h \cdot m}\left(f\left(X_{\zeta-}\right) ; \zeta \leqq t\right) & =\lim _{n \rightarrow \infty} E_{h \cdot m}\left(\sum_{k=1}^{\left[t / t_{n}\right]} f\left(X_{(k-1) t_{n}}\right) \cdot I_{\left\{(k-1) t_{n}<\zeta \leqq k t_{n}\right\}}\right) \\
& =\lim _{n \rightarrow \infty} \sum_{k=1}^{\left[t / t_{n}\right]} E_{h \cdot m}\left[f\left(X_{(k-1) t_{n}}\right)\left\{1-p_{t_{n}} 1\left(X_{(k-1) t_{n}}\right)\right\}\right] \\
& =\lim _{n \rightarrow \infty} \sum_{k=1}^{\left[t / t_{n}\right]}\left(p_{(k-1) t_{n}} h, f\left(1-p_{t_{n}} 1\right)\right) .
\end{aligned}
$$

Choose $\delta>0$ such that $\left\|p_{s} h-h\right\|_{L^{2}} \leqq 2 s\|A h\|_{L^{2}}$ for any $s<\delta$. For $t_{n}<\delta$, 


$$
\begin{aligned}
& \left|\sum_{k=1}^{\left[t / t_{n}\right]}\left(p_{(k-1) t_{n}} h, f\left(1-p_{t_{n}} 1\right)\right)-\frac{1}{t_{n}} \int_{0}^{t}\left(p_{s} h, f\left(1-p_{t_{n}} 1\right)\right) d s\right| \\
& \leqq \sum_{k=1}^{\left[t / t_{n}\right]} \frac{1}{t_{n}} \int_{(k-1) t_{n}}^{k t_{n}}\left|\left(p_{(k-1) t_{n}} h-p_{s} h, f\left(1-p_{t_{n}} 1\right)\right)\right| d s \\
& \quad+\frac{1}{t_{n}} \int_{t-\left[t / t_{n}\right]}^{t}\left|\left(p_{s} h, f\left(1-p_{t_{n}} 1\right)\right)\right| d s \leqq 2 t\|A h\|_{L^{2}} \cdot\left\|f\left(1-p_{\delta} 1\right)\right\|_{L^{2}} \\
& +\|h\|_{L^{2}} \cdot\left\|f\left(1-p_{\delta} 1\right)\right\|_{L^{2}},
\end{aligned}
$$

which decreases to zero as $\delta \downarrow 0$. Therefore

$$
E_{h \cdot m}\left(f\left(X_{\zeta-}\right) ; \zeta \leqq t\right)=\lim _{n \rightarrow \infty} \frac{1}{t_{n}} \int_{1}^{t}\left(p_{s} h, f\left(1-p_{t_{n}} 1\right)\right) d s=\int_{0}^{t}\left\langle p_{s} h \cdot f, k\right\rangle d s
$$

proving (7.2) for $f \in C_{0}^{+}(X)$ and $h=\alpha R_{\alpha} g, g \in C_{0}^{+}(X)$. Letting $\alpha$ tend to infinity, we know that (7.2) holds for any $f, h \in C_{0}^{+}(X)$.

q.e.d.

We define the resurrected Dirichlet form $\mathscr{E}^{\text {res }}$ by

$$
\mathscr{E}^{\operatorname{res}}(u, v)=\mathscr{E}(u, v)-\int_{X} \tilde{u}(x) \tilde{v}(x) k(d x), \quad u, v \in \mathscr{F}
$$

Then the formula (7.3) leads us to

$$
\mathscr{E}^{\mathscr{r} \mathrm{res}}(u, u)=\lim _{t \downarrow 0} \frac{1}{2 t} E_{m}\left(\left(u\left(X_{t}\right)-u\left(X_{0}\right)\right)^{2}\right), \quad u \in \mathscr{F}
$$

As an immediate consequence of Proposition 7.1, we have

Corollary. Following conditions are equivalent:

(i) there is no killing inside $X: P_{x}\left(X_{\zeta-} \in X, \zeta<\infty\right)=0$ q.e. $x \in X$.

(ii) $k=0$

(iii) $\mathscr{E}^{\mathrm{res}}(u, v)=\mathscr{E}(u, v), u, v \in \mathscr{F}$.

\section{(II) Transience and the extended Dirichlet space}

The Dirichlet space $\mathscr{F}$ is a real Hilbert space with inner product $\mathscr{E}_{1}$ but it is not even a pre-Hilbert space with respect to the 0 -order Dirichlet form $\mathscr{E}$ in general. We assert that $(\mathscr{F}, \mathscr{E})$ can be enlarged to an "extended Dirichlet space" relative to some weighted $L^{1}$-space if and only if the process $M$ is transient.

We say that the process $M$ is transient if

$$
R f(x)=\int_{0}^{\infty} p_{t} f(x) d t
$$


is finite $m$-a.e. for any non-negative Borel $f \in L^{1}(X ; m)$. By making use of Hopf's maximum ergodic inequality, we can see that $M$ is transient if and only if $R f(x)<\infty m$-a.e. for some strictly positive function $f \in L^{1}(X ; m)$.

A Dirichlet space $(\mathscr{F}, \mathscr{E})$ relative to $L^{2}(X ; m)$ is called transient if there exists a bounded strictly positive function $g \in L^{1}(X ; m)$ such that

$$
\int_{X}|u| g d m \leqq \sqrt{\mathscr{E}(u, u)} \quad \forall u \in \mathscr{F}
$$

The function $g$ above is called a reference function of the transient Dirichlet space $(\mathscr{F}, \mathscr{E})$.

Proposition 7.2. The process $M$ is transient if and only if the associated Dirichlet space $(\mathscr{F}, \mathscr{E})$ is transient.

This proposition can be proved by utilizing the identity

$$
\sup _{u \in \mathscr{F}} \frac{(|u|, g)}{\sqrt{\mathscr{E}(u, u)}}=\sqrt{\int_{X} g \cdot R g d m} \quad(\leqq \infty)
$$

holding for any non-negative $g \in L^{1} \cap L^{2}$.

A pair $\left(\mathscr{F}_{e}, \mathscr{E}\right)$ is called an extended Dirichlet space with reference measure $m$ if following conditions are satisfied:

$\left(\mathscr{F}_{e} \cdot 1\right) \mathscr{F}_{e}$ is a real Hilbert space with inner product $\mathscr{E}$, $\left(\mathscr{F}_{e}, 2\right)$ there exists an $m$-integrable bounded function $g$ strictly positive $m$-a.e. such that $\mathscr{F}_{e} \subset L^{1}(X ; g \cdot m)$ and inequality (7.8) holds for any $u \in \mathscr{F}_{e}$, $\left(\mathscr{F}_{e} \cdot 3\right) \quad \mathscr{F}_{e} \cap L^{2}(X ; m)$ is dense both in $L^{2}(X ; m)$ and in $\left(\mathscr{F}_{e}, \mathscr{E}\right)$, $\left(\mathscr{F}_{e} \cdot 4\right)$ every normal contraction operates on $\left(\mathscr{F}_{e}, \mathscr{E}\right)$.

Proposition 7.2. Assume that the process $M$ (and consequently the associated Dirichlet space $(\mathscr{F}, \mathscr{E}))$ is transient.

(i) The completion $\left(\mathscr{F}_{e}, \mathscr{E}\right)$ of $(\mathscr{F}, \mathscr{E})$ is an extended Dirichlet space with reference measure $m$. $\mathscr{F}=\mathscr{F}_{e} \cap L^{2}(X ; m)$.

(ii) For any Borel $u \in \mathscr{F}_{e}$, the left hand side of (7.3) increases as $t \downarrow 0$ to $\mathscr{E}(u, u)$.

The ideas of the proof are found in [1] and [12]. Since $(\mathscr{F}, \mathscr{E})$ is assumed to be regular, so is the extended Dirichlet space $\left(\mathscr{F}_{e}, \mathscr{E}\right)$ of Proposition 7.2 in the following sense:

$\left(\mathscr{F}_{e} \cdot 3\right)^{\prime} \quad \mathscr{F}_{e} \cap C_{0}(X)$ is dense both in $\left(\mathscr{F}_{e}, \mathscr{E}\right)$ and $C_{0}(X)$.

Accordingly each $u \in \mathscr{F}_{e}$ has a quasi-continuous version $\tilde{u}$ ([12]). Propo- 
sition 7.2 (ii) then implies that (7.1) extends to any $u \in \mathscr{F}_{e}$. Moreover if we define $\mathscr{E}^{\text {res }}(u, v)$ for $u, v \in \mathscr{F}_{e}$ by (7.5), then the identity (7.6) extends to $u \in \mathscr{F}_{e}$.

\section{REFERENCES}

[1] J. Deny, Méthodes hilbertiennes en theory du potential, Potential Theory, C.I.M.E., Edizioni Cremonese, Roma, 1970.

[2] M. Fukushima, On the generation of Markov processes by symmetric forms, Proc. 2nd Japan-USSR Symp. on Prob. Th., Lecture Notes in Math. 330, SpringerVerlag, Berlin-Heidelberg-New York, 1973.

[ 3 ] M. Fukushima, Potential theory of symmetric Markov processes and its applications, Proc. 3rd Japan-USSR Symp, on Proc. Th., Lecture Notes in Math. 550, Springer-Verlag, Berlin-Heidelberg-New York, 1976.

[4] M. Fukushima, On additive functionals admitting exceptional sets, to appear in J. Math. Kyoto Univ.

[5] R. K. Getoor and M. J. Sharpe, An extension of Ito's formula, Preprint.

[6] H. Kunita and S. Watanabe, On square integrable martingales, Nagoya Math. J., 30 (1967), 209-245.

[ 7 ] N. V. Krylov, On Ito's stochastic integral equations, Theory Prob. Applications, 14 (1969), 330-336.

[ 8 ] Yves LeJan, Mesures associées a une forme de Dirichlet-Applications, Bull. Soc. Math. France 106 (1978), 61-112.

[ 9 ] H. P. McKean, Stochastic integrals, Academic press, New York, 1969.

[10] P. A. Meyer, Intégrales stochastiques I, II, III, Seminaire de Probabilités, 1, Lecture Notes in Math. 39, Springer-Verlag, Berlin-Heidelbert-New York, 1967.

[11] M. Motoo and S. Watanabe, On a class of additive functionals of Markov processes, J. Math. Kyoto Univ. 4 (1965), 429-469.

[12] M. L. Silverstein, Symmetric Markov processes, Lecture Notes in Math. 426, Springer-Verlag, Berlin-Heidelberg-New York, 1974.

[13] M. Tsuchiya, On an extension of Ito's formula, Ann. Sci. Kanazawa Univ. 14 (1977), 7-13.

College of General Education

Osaka University 